\title{
Comparison of two sampling schemes in estimating the stress-strength reliability under the proportional reversed hazard rate model
}

\author{
Amineh Sadeghpour ${ }^{1}$, Ahmad Nezakati ${ }^{1, *}$, Mahdi Salehi ${ }^{2}$ \\ ${ }^{1}$ Faculty of Mathematical Sciences, Shahrood University of Technology, Iran \\ ${ }^{2}$ Department of Mathematics and Statistics, University of Neyshabur, Neyshabur, Iran
}

\begin{abstract}
In this paper, point and interval estimation of stress-strength reliability based on lower record ranked set sampling (RRSS) scheme under the proportional reversed hazard rate model are considered. Maximum likelihood, uniformly minimum variance unbiased estimator, and Bayesian estimators of $\mathcal{R}$ are derived. Also, we compared this point estimators with their counterparts obtained by well-known sampling scheme in record values known as inverse sampling scheme. Various confidence intervals for the parameter $\mathcal{R}$ are constructed, and compared based on the simulation study. Moreover, the RRSS scheme is compared with ordinary records in case of interval estimations. We observed that our proposed point and interval estimations perform well in the estimation of $\mathcal{R}$ based on RRSS. We also proved that all calculations do not depend on the baseline distribution in the proportional reversed hazard rate model. Finally, a data set has been analyzed for illustrative purposes.
\end{abstract}

Keywords Record values, Relative efficiency, Bootstrap confidence interval, Maximum likelihood estimator, Proportional reversed hazard rate model, Record ranked set sampling, Stress-Strength reliability, Uniformly minimum variance unbiased estimator.

AMS 2010 subject classifications 62F10, 62G30

DOI: $10.19139 /$ soic-2310-5070-781

\section{Introduction}

The stress-strength reliability is defined as $\mathcal{R}:=\operatorname{Pr}(X<Y)$ or the probability of not failing when a random stress $X$ is applied to a certain device with strength $Y$. For example, the random variable $Y$ represents the strength of a building during an earthquake and the random variable $X$ is stress from the earthquake. Therefore, $\mathcal{R}$ could be defined as the probability of designing for a safe building. Some practical examples could be found in Kotz et al. [15]. Many authors have studied the stress-strength parameter $\mathcal{R}$ in parametric and non-parametric inferences by considering several specific distributions of $X$ and $Y$ under different sampling schemes. There are several studies on the estimation of $\mathcal{R}$ based on the complete samples such as Raqab et al. [20] and Rezaei et al. [21] who estimated $\mathcal{R}$ for the three-parameter Weibull and generalized Pareto distributions, respectively. Also, Mutllak et al. [17] considered estimation of $\mathcal{R}$ using ranked set sampling (RSS) in the case of the exponential distribution. Recently, some authors have investigated the statistical inference for $\mathcal{R}$ based on record data. Baklizi [4] studied the likelihood and Bayesian estimations of $\mathcal{R}$ using lower record values from the generalized exponential distribution (GE). Asgharzadeh et al. [2] obtained the estimation of $\mathcal{R}$ for the two-parameter generalized exponential records. Moreover, Basirat et al. [5] estimated the stress-strength parameter for the proportional hazard rate (PHR) models based on record values. Let $X_{1}, X_{2}, \ldots$, be an infinite sequence of independent and identically distributed (iid)

\footnotetext{
*Correspondence to: Ahmad Nezakati (Email: nezakati@shahroodut.ac.ir). Faculty of Mathematical Sciences, Shahrood University of Technology, Shahrood, Iran.
}

ISSN 2310-5070 (online) ISSN 2311-004X (print)

Copyright (C) 2021 International Academic Press 
random variables. An observation $X_{j}$ is called a lower record if $X_{j}<X_{i}$ for every $(i<j)$. Salehi and Ahmadi [25] introduced a new sampling scheme in record-breaking data. They proposed that more accurate results can be achieved for compared ordinary record values. Suppose $n$ independent sequences are considered sequentially, and the $i$ th sequence sampling is terminated when the $i$ th record is observed. The only available observations for analysis are the last record value in each sequence. Let us denote the last record for the $i$ th sequence in this plan by $L_{i, i}$. Then the available observations are $\mathbf{L}=\left(L_{1,1}, L_{2,2}, \ldots, L_{n, n}\right)^{\top}$. Therefore, the following diagram can be used to describe this observational process:

$$
\begin{aligned}
& 1: L_{(1) 1} \\
& 2: L_{(1) 2} L_{(2) 2} \\
& \rightarrow L_{1,1}=L_{(1) 1} \\
& \rightarrow L_{2,2}=L_{(2) 2} \\
& \vdots \quad \vdots \quad \vdots \cdot \\
& n: L_{(1) n} L_{(2) n} \quad \cdots L_{(n) n} \\
& \rightarrow L_{n, n}=L_{(n) n},
\end{aligned}
$$

Where $L_{(i) i}$ is the $i$ th ordinary lower record in the $i$ th sequence. Notice that unlike the ordinary records, here $L_{i, i}$ 's are independent random variables but not necessarily ordered. The proposed scheme is called RRSS because this scheme is based on general ranked set sampling. Let $F(\cdot ; \theta)$ and $f(\cdot ; \theta)$ be the cumulative distribution function (cdf) and probability density function (pdf) of the sampling population, respectively. Then, using the marginal density of ordinary record (see Arnold et al. [1]) the joint density of $\mathbf{L}$ is readily obtained as

$$
f_{\mathbf{L}}(\mathbf{l} ; \theta)=\prod_{i=1}^{n} \frac{\left\{-\log F\left(l_{i, i} ; \theta\right)\right\}^{i-1}}{(i-1) !} f\left(l_{i, i} ; \theta\right), \quad \theta \in \Theta,
$$

where $\mathbf{l}=\left(l_{1,1}, l_{2,2}, \ldots, l_{n, n}\right)^{\top}$ is the observed vector of $\mathbf{L}, \theta$ is a real-valued parameter and $\Theta$ is the parameter space. For the upper case, we use $\bar{F}$ instead of $F$ where $\bar{F}=1-F$.

Salehi and Ahmadi [26] considered the estimation of stress-strength reliability based on RRSS for the exponential distribution. With collaboration of Dey [27], they also compared the RRSS scheme with ordinary records in estimating the unknown parameter of PHR model. The RRSS plan has also been investigated by other researchers. Among them, Eskandarzadeh et al. [11] obtained information measure for RRSS. Paul and Thomas [18] proposed concomitant RRSS. Safariyan et al. [23, 24] proposed some improved estimators including the preliminary test estimator as well as stein- type shrinkage estimator for stress-strength reliability using RRSS scheme. Sadeghpour et al. [22] considered the likelihood and Bayesian estimation of stress-strength reliability using lower RRSS scheme under the GE distribution.

The concept of proportional reversed hazard rate (PRHR) models (see Gupta et al. [13]) has been extensively studied in literature because of their wide applicability in survival analysis and reliability, especially in studies on parallel systems and in analysis of left-censored lifetime data, (see Sengupta and Nanda [28] and Chandra and Roy [8]). The PRHR model is equivalent to the model $G(t)=\left[F_{0}(t)\right]^{\theta}$, where $F_{0}(t)$ is the baseline distribution function which is free of any unknown parameter. For more applications of reversed hazard rate, see Gupta and Nanda [12] and Gupta and Gupta [14].

It is known that many lifetime models, namely the generalized exponential, Weibull, Rayleigh, Pareto, logistic, inverse Rayleigh, Bur type III, exponential Gumbel and power distributions belong to the class of PRHR distributions. Moreover, the stress-strength reliability has received considerable attention in branches of sciences such as industry, medicine, and engineering, and the necessity of using the appropriate method for collecting observations. Thus, to achieve high reliability in the shortest time and at a minimum cost based on the RRSS, the main objective of this paper is to make a statistical inference for stress-strength reliability based on lower RRSS scheme where $X$ and $Y$ are two independent random variables that follow the PRHR model. In fact, we compare the performance of stress-strength reliability based on two scheme in record-breaking data, one of which is inverse sampling scheme and other scheme is RRSS.

The rest of the paper is organized as follow: In Section 2, the maximum likelihood (MLE) and uniformly minimum variance unbiased (UMVUE) as well as the Bayesian estimator of $\mathcal{R}$ are derived, and their performance is 
compared with their counterparts obtained by inverse sampling scheme in record values. Various confidence intervals (CI) as well as Bayesian credible interval and highest posterior density (HPD) for $\mathcal{R}$ are presented in Section 3. In Section 4, analysis of a simulated data set has been presented, and the results of interval estimations are compared with those obtained based on ordinary records. Finally, a real data set on the time of successive failures of air conditioning system in Boeing 720 jet airplanes is analyzed in section 5.

\section{Point Estimation}

Let us recall that the continuous random variable $\mathrm{T}$ has the cdf and pdf in the form of

$$
G(t)=\left[F_{0}(t)\right]^{\theta} \text { and } g(t)=\theta f_{0}(t)\left[F_{0}(t)\right]^{\theta-1},
$$

respectively, where $F_{0}(t)$ is the baseline cdf which is independent of the shape parameter $\theta$ where $\theta>0$, this family of distribution is well known as PRHR model. Let $X$ and $Y$ be two independent random variables following PRHR model with the parameter $\alpha$ and $\beta$, respectively. It is easy to see that in this case $\mathcal{R}=\operatorname{Pr}(X<Y)=\frac{\beta}{\alpha+\beta}$. We are interested in estimating the stress-strength $\mathcal{R}$ when the samples are permitted to be a lower RRSS's with possibly different number of observations. Let $\mathbf{u}=\left(u_{1,1}, u_{2,2}, \cdots, u_{n, n}\right)^{\top}$ be the observation of the random vector $\mathbf{U}=\left(U_{1,1}, U_{2,2}, \cdots, U_{n, n}\right)^{\top}$, which is a lower RRSS of size $n$ from PRHR models with cumulative distribution function $G(u)=\left(F_{0}(u)\right)^{\alpha}$. Also let $\mathbf{s}=\left(s_{1,1}, s_{2,2}, \cdots, s_{m, m}\right)^{\top}$ be the observation of random vector $\mathbf{S}=\left(S_{1,1}, S_{2,2}, \cdots, S_{m, m}\right)^{\top}$, which is a lower RRSS of size $m$ from PRHR model with cumulative distribution function $G(s)=\left(F_{0}(s)\right)^{\beta}$.

For estimating $\mathcal{R}$ via maximum likelihood method, it is necessary to obtain the MLE's of $\alpha$ and $\beta$. By using (2), the likelihood function will be obtained as

$$
\begin{aligned}
L(\alpha, \beta) & =\prod_{i=1}^{n}\left\{\frac{\left\{-\log \left(F\left(u_{i, i}\right)^{\alpha}\right)\right\}^{i-1}}{(i-1) !} \alpha f\left(u_{i, i}\right)\left[F\left(u_{i, i}\right)^{\alpha-1}\right]\right\} \\
& \times \prod_{j=1}^{m}\left\{\frac{\left\{-\log \left(F\left(s_{i, i}\right)^{\beta}\right)\right\}^{j-1}}{(j-1) !} \beta f\left(s_{j, j}\right)\left[F\left(s_{j, j}\right)^{\beta-1}\right]\right\},
\end{aligned}
$$

Therefore, the MLE's of $\alpha$ and $\beta$ are immediately obtained as

$$
\hat{\alpha}_{M L}=-\frac{N}{\sum_{i=1}^{n} \log \left(F\left(U_{i, i}\right)\right)} \quad \text { and } \quad \hat{\beta}_{M L}=-\frac{M}{\sum_{j=1}^{m} \log \left(F\left(S_{j, j}\right)\right)},
$$

where $N=\frac{n(n+1)}{2}$ and $M=\frac{m(m+1)}{2}$.

Consequently, $\hat{\mathcal{R}}_{M L}$ could be defined by using invariance property .

$$
\hat{\mathcal{R}}_{M L}=\frac{\hat{\beta}_{M L}}{\hat{\alpha}_{M L}+\hat{\beta}_{M L}}=\left(1+\frac{N H_{m}^{\prime}}{M T_{n}^{\prime}}\right)^{-1},
$$

where $T_{n}^{\prime}=-\sum_{i=1}^{n} \log \left(F\left(U_{i, i}\right)\right)$ and $H_{m}^{\prime}=-\sum_{j=1}^{m} \log \left(F\left(S_{j, j}\right)\right)$. As discussed before, $U_{i, i}$ 's and $S_{j, j}$ 's are independent random variables. Moreover, it can be shown that $T_{i}=-\log \left(F\left(U_{i, i}\right)\right)$ where $i=1, \ldots, n$ follows from Gamma distribution with the shape parameter $i$ and the scale parameter $\alpha$, denoted by $T_{i} \sim \operatorname{Gamma}(i, \alpha)$. Therefore, we have

$$
T_{n}^{\prime}=-\sum_{i=1}^{n} \log \left(F\left(U_{i, i}\right)\right) \sim \operatorname{Gamma}(N, \alpha),
$$


and similarly

$$
H_{m}^{\prime}=-\sum_{j=1}^{m} \log \left(F\left(S_{j, j}\right)\right) \sim \operatorname{Gamma}(M, \beta) .
$$

From (6) and (7) clearly, we have

$$
\hat{\mathcal{R}}_{M L} \stackrel{d}{=}\left(1+\frac{\alpha}{\beta} W\right)^{-1}
$$

where $\stackrel{d}{=}$ means equivalent in distribution and $W$ has F-distribution with $2 M$ and $2 N$ degrees of freedom. According to (8) and the results obtained in [22], it is observed that the distribution of $\hat{\mathcal{R}}_{M L}$ based on RRSS scheme from the PRHR model does not depend on the baseline distribution, $F_{0}$.

We use (8) for obtaining $M S E$ of $\hat{\mathcal{R}}_{M L}$, i.e. $\operatorname{Biase}\left(\hat{\mathcal{R}}_{M L}, \mathcal{R}\right)=E\left(\hat{\mathcal{R}}_{M L}-\mathcal{R}\right)$ and $M S E\left(\hat{\mathcal{R}}_{M L}, \mathcal{R}\right)=$ $E\left(\hat{\mathcal{R}}_{M L}-\mathcal{R}\right)^{2}$, respectively. In Figure 1, we have plotted the values of $M S E$ and Bias of $\hat{\mathcal{R}}_{M L}$ versus $\mathcal{R}$ for some selected values of $n$ and $m$.

According to Figure 1, we could observe the following points:

- When $n=m$, the $\operatorname{MSE}\left(\hat{\mathcal{R}}_{M L}, \mathcal{R}\right)$ and $\operatorname{Bias}\left(\hat{\mathcal{R}}_{M L}, \mathcal{R}\right)$ are symmetric around $\mathcal{R}=0.5$ and about the point $(0.5,0)$ respectively. Also, the Bias only is zero for $\mathcal{R}=0,0.5,1$. [see part (a) and (b) ].

- According to part (c) when $n \neq m$, the curve of $\operatorname{MSE}\left(\hat{\mathcal{R}}_{M L}, \mathcal{R}\right)$ is asymmetric. When $n<m$, the curve has skewness to the right and when $n>m$, it has skewness to the left. Furthermore, when $n=m$ the $M S E\left(\hat{\mathcal{R}}_{M L} ; \mathcal{R}\right)$ is significantly less than the case where $n \neq m$.

- When the sample sizes increase, the $\operatorname{MSE}\left(\hat{\mathcal{R}}_{M L}, \mathcal{R}\right)$ decreases, and we observe that $\mathcal{R}_{\text {max }} \propto \frac{n}{n+m}$, where $\mathcal{R}_{\text {max }}$ is the maximizer of $M S E\left(\hat{\mathcal{R}}_{M L}, \mathcal{R}\right)$.

Now, we obtain UMVUE of $\mathcal{R}$ based on RRSS scheme from the PRHR model.

\section{Lemma 1}

Let the random vector $\mathbf{U}=\left(U_{1,1}, \ldots, U_{n, n}\right)^{T}$ and $\mathbf{S}=\left(S_{1,1}, \ldots, S_{m, m}\right)^{T}$ be lower RRSS of size $n$ and $m$ from PRHR models respectively. Then, the UMVUE of $\mathcal{R}$ is derived as follows.

$$
\hat{\mathcal{R}}_{U M V U}=\left\{\begin{array}{c}
\sum_{s=0}^{M-1} \frac{\left(\begin{array}{c}
M-1 \\
s
\end{array}\right)}{\left(\begin{array}{c}
N+s-1 \\
s
\end{array}\right)}\left(-\frac{1-\hat{\mathcal{R}}_{M L}}{\hat{\mathcal{R}}_{M L}} \frac{M}{N}\right)^{s}, \quad \hat{\mathcal{R}}_{M L}<\frac{M}{M+N}, \\
1-\sum_{s=0}^{N-1} \frac{\left(\begin{array}{c}
N-1 \\
s
\end{array}\right)}{\left(\begin{array}{c}
M+s-1 \\
s
\end{array}\right)}\left(-\frac{\hat{\mathcal{R}}_{M L}}{1-\hat{\mathcal{R}}_{M L}} \frac{N}{M}\right)^{s}, \quad \hat{\mathcal{R}}_{M L} \geq \frac{M}{M+N} .
\end{array}\right.
$$

For more details see [22]. According to (9), the UMVUE of $\mathcal{R}$ is derived in term of the $\hat{\mathcal{R}}_{M L}$. So, $\hat{\mathcal{R}}_{U M V U}$ based on RRSS scheme from the PRHR model does not depend on the baseline distribution, $F_{0}$.

By using (9), we have plotted the numerical values of $\operatorname{Var}\left(\hat{\mathcal{R}}_{U M V U}\right)$ versus $\mathcal{R}$ for some selected values of $n$ and $m$ show in Figure 2.

We observe that when $n=m$, the behaviour of $\operatorname{Var}\left(\hat{\mathcal{R}}_{U M V U}\right)$ is almost similar to $\operatorname{MSE}\left(\hat{\mathcal{R}}_{M L}, \mathcal{R}\right)$ but when $n \neq m$ it has a different behaviour and departures from symmetry.

With respect to (9), we plot the numerical values of the estimators $\hat{\mathcal{R}}_{M L}$ and $\hat{\mathcal{R}}_{U M V U}$ against each other in Figure 3. We observe that when $n=m, \hat{\mathcal{R}}_{M L}$ and $\hat{\mathcal{R}}_{U M V U}$ hold values close together but when $n \neq m$, they have different values. So, to compare the effectiveness of this point estimators, the numerical values of the efficiency of these estimators i.e.

$$
\mathbf{e}\left(\hat{\mathcal{R}}_{U M V U}, \hat{\mathcal{R}}_{M L}\right)=\frac{\operatorname{Var}\left(\hat{\mathcal{R}}_{U M V U}\right)}{M S E\left(\hat{\mathcal{R}}_{M L}, \mathcal{R}\right)} .
$$




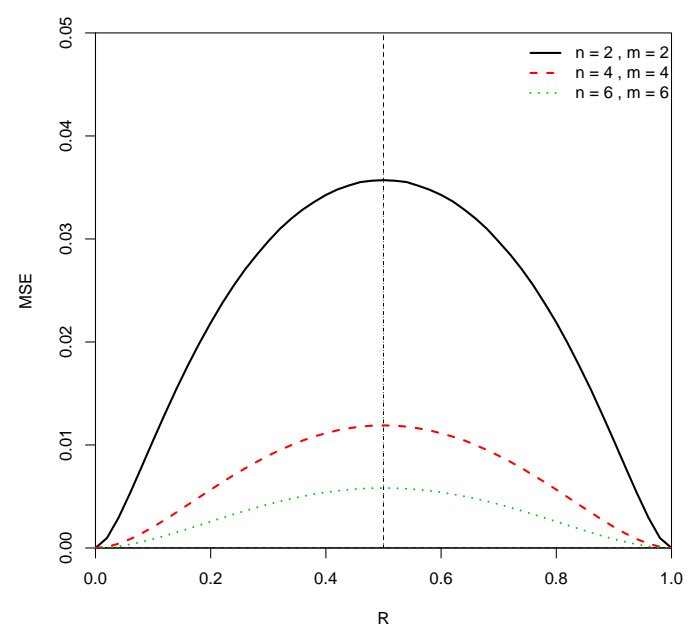

(a)

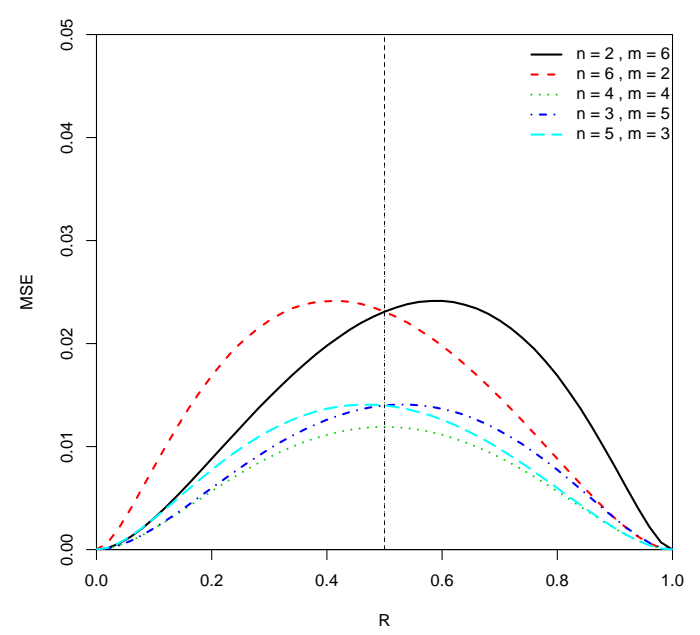

(c)

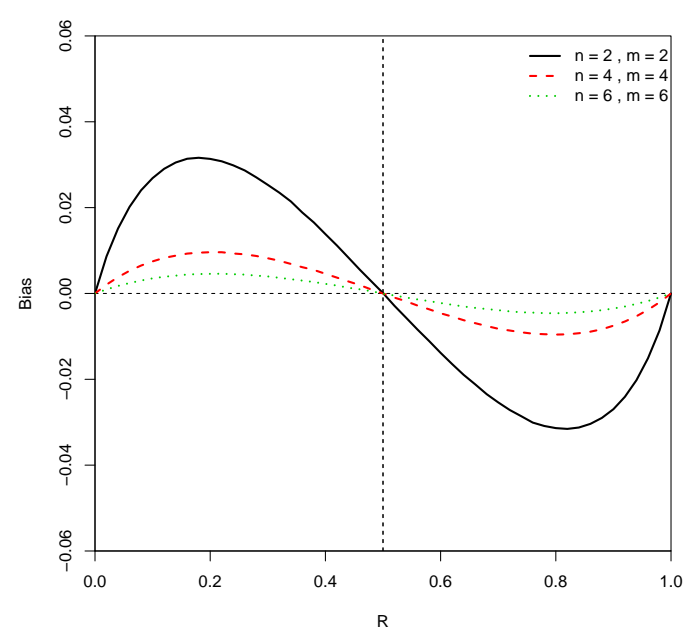

(b)

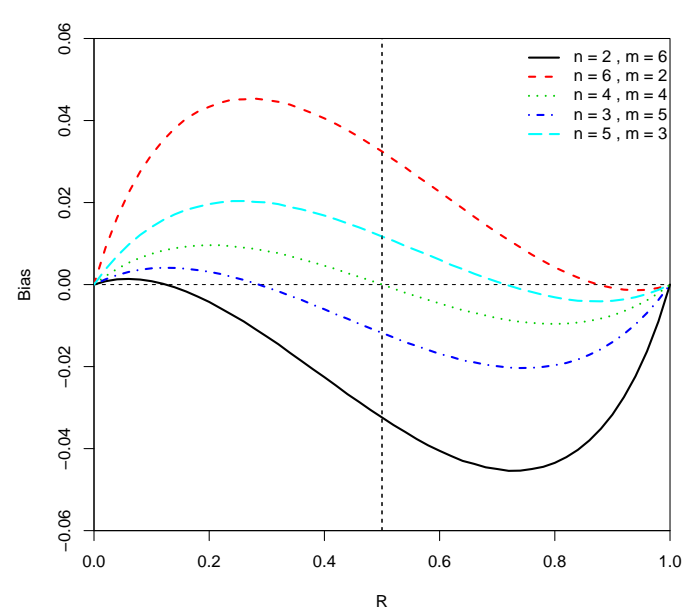

(d)

Figure 1. Plot of $M S E\left(\hat{\mathcal{R}}_{M L}, \mathcal{R}\right)$ and $\operatorname{Bias}\left(\hat{\mathcal{R}}_{M L}, \mathcal{R}\right)$ versus $\mathcal{R}$.

is plotted in Figure 4.

We observe from the figure that when $n=m$, the relative efficiency of $\hat{\mathcal{R}}_{M L}$ is more than the relative efficiency of $\hat{\mathcal{R}}_{U M V U}$ especially for the values of $\mathcal{R}$ near the half.

\subsection{Bayesian estimation}

In this section, we focus in Bayesian estimation of $\mathcal{R}$ under the assumptions that the $\alpha$ and $\beta$ have independent gamma prior, i.e., $\alpha \sim \operatorname{Gamma}\left(\lambda_{1}, \mu_{1}\right)$ and $\beta \sim \operatorname{Gamma}\left(\lambda_{2}, \mu_{2}\right)$. Thus the posterior distribution of $\alpha$ and $\beta$ are

$$
\alpha \mid \mathbf{r} \sim \operatorname{Gamma}\left(\lambda_{1}+N, \mu_{1}+t_{n}^{\prime}\right), \text { and } \beta \mid \mathbf{s} \sim \operatorname{Gamma}\left(\lambda_{2}+M, \mu_{2}+h_{m}^{\prime}\right),
$$



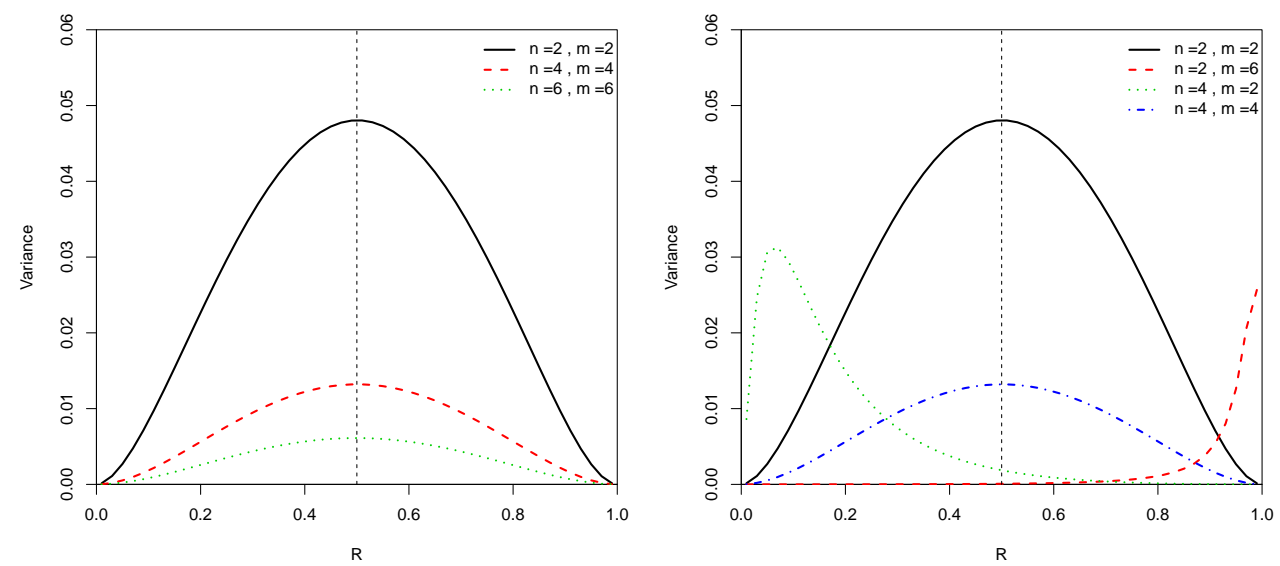

Figure 2. Plot of $\operatorname{Var}\left(\hat{\mathcal{R}}_{U M V U}\right)$ versus $\mathcal{R}$.

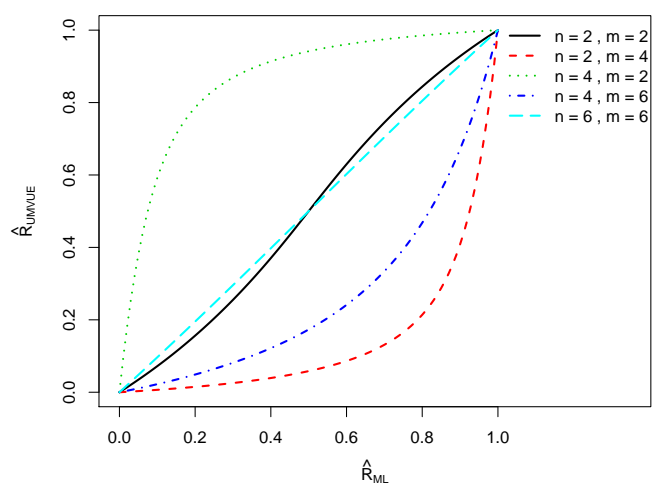

Figure 3. Plot of $\hat{\mathcal{R}}_{U M V U}$ versus $\hat{\mathcal{R}}_{M L}$.

where $t_{n}^{\prime}$ and $h_{m}^{\prime}$ are the observed values of random variables $T_{n}^{\prime}$ and $H_{m}^{\prime}$ in (6) and (7) respectively. Suppose that $\alpha$ and $\beta$ are independent, so the posterior distribution of $\mathcal{R}$ is given by

$$
f_{\mathcal{R} \mid(\mathbf{R}, \mathbf{S})}(r)=\frac{\Gamma\left(\lambda_{1}+\lambda_{2}+N+M\right)}{\Gamma\left(\lambda_{1}+N\right) \Gamma\left(\lambda_{2}+M\right)}\left(\frac{\mu_{2}+H_{m}^{\prime}}{\mu_{1}+T_{n}^{\prime}}\right)^{\lambda_{2}+M} \frac{r^{\lambda_{2}+M-1}(1-r)^{\lambda_{1}+N-1}}{\left(1-r\left(1-\frac{\mu_{2}+H_{m}^{\prime}}{\mu_{1}+T_{n}^{\prime}}\right)\right)^{\lambda_{1}+\lambda_{2}+N+M}} .
$$

The Bayesian estimator of $\mathcal{R}$ under squared error loss function will be derived as (see [7]).

$$
\begin{aligned}
\hat{\mathcal{R}}_{B} & =\int_{0}^{1} r f_{\mathcal{R} \mid(\mathbf{R}, \mathbf{S})}(r) d r=\frac{\lambda_{2}+M}{\lambda_{1}+\lambda_{2}+N+M}\left(\frac{\mu_{2}+H_{m}^{\prime}}{\mu_{1}+T_{n}^{\prime}}\right)^{\lambda_{2}+M} \\
& \times{ }_{2} F_{1}\left(\lambda_{1}+\lambda_{2}+N+M, \lambda_{2}+M+1, \lambda_{1}+\lambda_{2}+N+M+1,\left(1-\frac{\mu_{2}+H_{m}^{\prime}}{\mu_{1}+T_{n}^{\prime}}\right)\right) .
\end{aligned}
$$

where ${ }_{2} \mathcal{F}_{1}$ is the hyper-geometric function, (see [3]). 


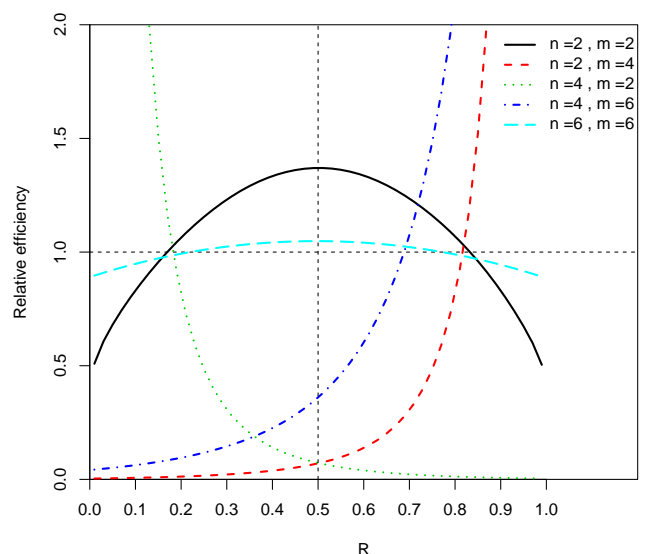

Figure 4. Plot of e( $\left.\hat{\mathcal{R}}_{U M V U} ; \hat{\mathcal{R}}_{M L}\right)$ versus $\mathcal{R}$.

\section{Comparison of point estimators of $\mathcal{R}$ based on two sampling schemes}

In section 2, we presented some point estimators for $\mathcal{R}=\operatorname{Pr}(X<Y)$ based on lower RRSS scheme for PRHR model. Also, Baklizi [4] considered the likelihood and Bayesian estimation of $\mathcal{R}$ based on lower record values from the GE distribution.

In this section, we compare the point estimators of $\mathcal{R}$ based on ordinary records (see [4]) with those obtained by the RRSS scheme. In inverse sampling scheme, the experiments are done sequentially, the only observation available for analysis is the record values and sampling is terminated when the $n$th record is observed. Let $X$ and $Y$ be two independent random variables from GE distribution with the parameter $\alpha$ and $\beta$, respectively. Also, let $u^{(R)}=\left(u_{1}, \ldots, u_{n^{\prime}}\right)^{\top}$ be the observed value of the random vector $U^{(R)}=\left(U_{1}, \ldots, U_{n^{\prime}}\right)^{\top}$ which is a lower record value of size $n^{\prime}$ from GE distribution and $s^{(R)}=\left(s_{1}, \ldots, s_{m^{\prime}}\right)^{\top}$ be the observed value of the random vector $S^{(R)}=\left(S_{1}, \ldots, S_{m^{\prime}}\right)^{\top}$, which is a lower record value of size $m^{\prime}$ from GE distribution. It can be shown that under this case, $\mathcal{R}=\frac{\beta}{\alpha+\beta}$.

- MLE: Note that by (8), we find that the $\operatorname{MSE}\left(\hat{\mathcal{R}}_{M L}, \mathcal{R}\right)$ does not depend on the distribution of the baseline random variable, $F_{0}$. So, we consider GE distribution as a special case of the PRHR model. we recall that the pdf of the GE distribution is given by

$$
f_{0}(t)=\theta e^{-t}\left(1-e^{-t}\right)^{\theta}, \quad t>0, \quad \theta>0 .
$$

To compare the MLE estimators of the two schemes, one of which is inverse sampling scheme and other scheme is RRSS, we use the relative efficiency criterion, i.e.

$$
\mathbf{e}\left(\hat{\mathcal{R}}_{M L}^{(R R S S)}, \hat{\mathcal{R}}_{M L}^{(R)}\right)=\frac{M S E\left(\hat{\mathcal{R}}_{M L}^{(R)}, \mathcal{R}\right)}{M S E\left(\hat{\mathcal{R}}_{M L}^{(R R S)}, \mathcal{R}\right)} .
$$

It is plotted in Figure 5 with considering equal sample sizes for two schemes. We observe from that figure, for all combination of $(n, m)$, the efficiency of $\hat{\mathcal{R}}_{M L}^{(R R S S)}$ is more than the $\hat{\mathcal{R}}_{M L}^{(R)}$. Also, this figure shows that when the sample sizes increase, the efficiency of $\hat{\mathcal{R}}_{M L}^{(R R S S)}$ is significantly better than $\hat{\mathcal{R}}_{M L}^{(R)}$.

Also, to be more focused on the comparison of the estimators, we have plotted the values of (14) with respect to 


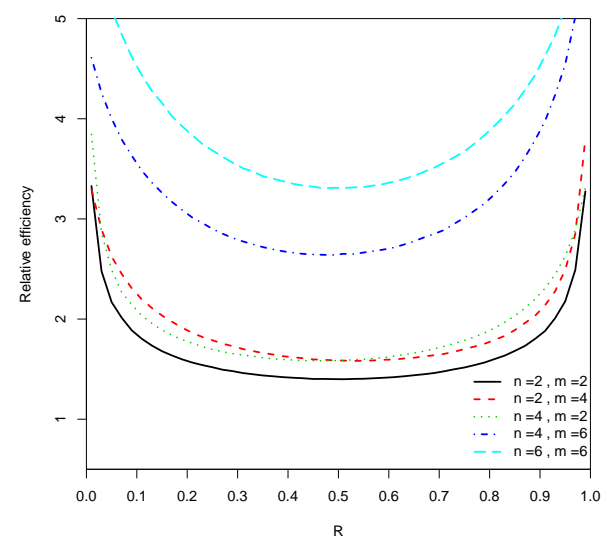

Figure 5. Plot of $\mathbf{e}\left(\hat{\mathcal{R}}_{M L}^{(R)} ; \hat{\mathcal{R}}_{M L}^{(R R S S)}\right)$ versus $\mathcal{R}$.

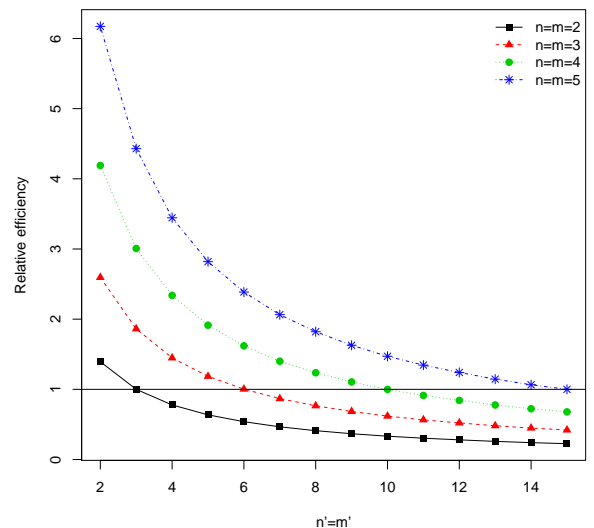

$\mathcal{R}=0.5$

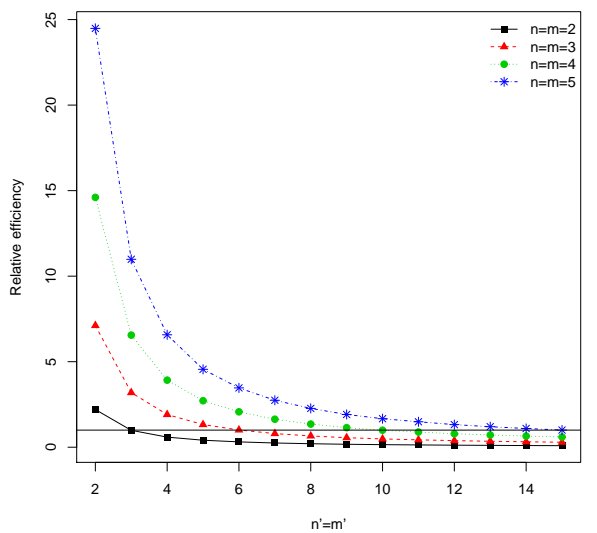

$\mathcal{R}=0.95$

Figure 6. The relative efficiency of $\hat{\mathcal{R}}_{M L}$ given in (14).

$n^{\prime}=m^{\prime}=2(1) 15$ for a given $n=m=2(1) 5$ for $\mathcal{R}=0.5,0.95$ in Figure 6.

From Figure 6, it is easy to observe that for $n^{\prime}=m^{\prime} \leq N=M$, the relative efficiency is greater than one. More clearly, when $n^{\prime} \leq N$ and $m^{\prime} \leq M$, the $\hat{\mathcal{R}}_{M L}^{(R R S S)}$ behaves better than $\hat{\mathcal{R}}_{M L}^{(R)}$. For example $\hat{\mathcal{R}}_{M L}^{(R R S S)}$ with $n=m=4$ (or equivalently $\mathrm{N}=\mathrm{M}=10$ ) is more efficient than the estimator $\hat{\mathcal{R}}_{M L}^{(R)}$ with $n^{\prime}=m^{\prime}=8$.

-UMVUE: The UMVUE of $\mathcal{R}$ is derived in terms of the MLE as equation (9). Note that by (8), we find that $\operatorname{Var}\left(\hat{\mathcal{R}}_{U M V U}\right)$ does not depend on the underlying distribution function of $X$ and $Y$. So, we consider GE distribution in (13) as a distribution of $X$ and $Y$. To compare the effectiveness of $\hat{\mathcal{R}}_{U M V U}$ with considering equal sample sizes for two schemes, the numerical values of the efficiency of these estimators i.e.

$$
\mathbf{e}\left(\hat{\mathcal{R}}_{U M V U}^{(R R S S)}, \hat{\mathcal{R}}_{U M V U}^{(R)}\right)=\frac{\operatorname{Var}\left(\hat{\mathcal{R}}_{U M V U}^{(R)}\right)}{\operatorname{Var}\left(\hat{\mathcal{R}}_{U M V U}^{(R R S S)}\right)}
$$


is plotted in Figure 7.

We observe from the figure that the UMVUE based on RRSS scheme is more efficient than the UMVUE on the

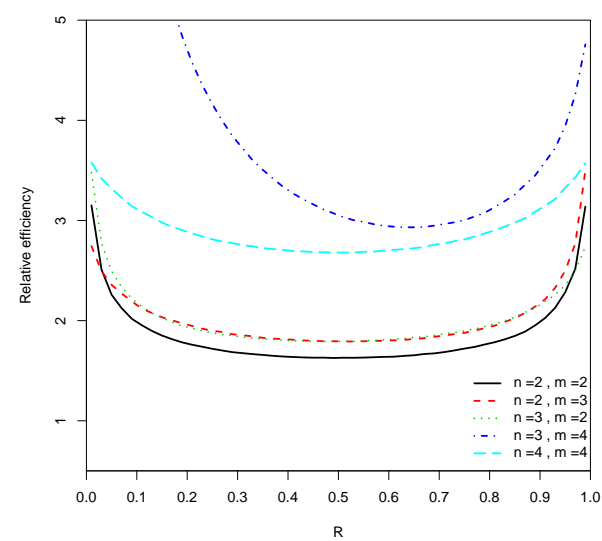

Figure 7. Plot of $\mathbf{e}\left(\hat{\mathcal{R}}_{U M V U}^{(R)} ; \hat{\mathcal{R}}_{U M V U}^{(R R S S)}\right)$ versus $\mathcal{R}$.

basis of ordinary record.

Similar to ML estimator, the values of relative efficiency in (15), with respect to $n^{\prime}=m^{\prime}=2(1) 15$ for a given $n=m=2(1) 5$ for $\mathcal{R}=0.5,0.95$ are plotted in Figure 8 .

We observe from the Figure 8 that the performance of $\hat{\mathcal{R}}_{U M V U}^{(R)}$ is better than $\hat{\mathcal{R}}_{U M V U}^{(R R S S)}$ when the number of sample

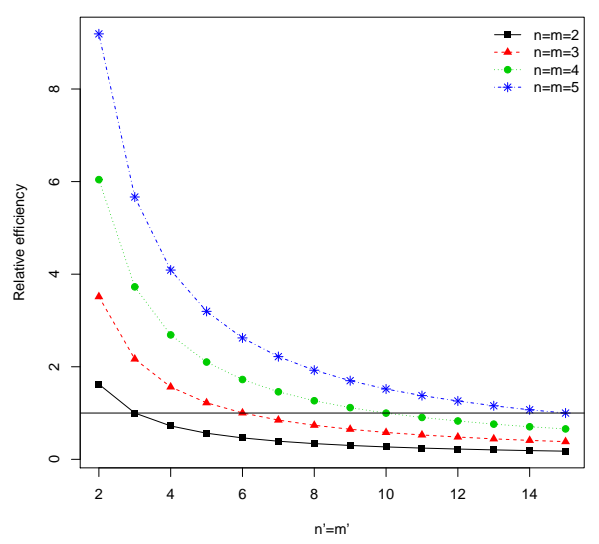

$\mathcal{R}=0.5$

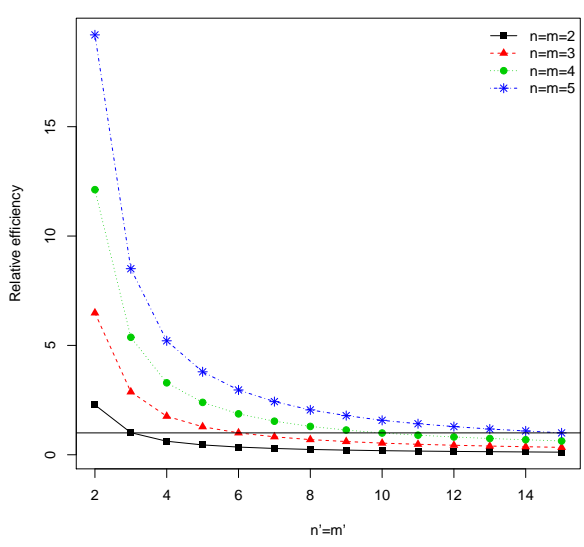

$\mathcal{R}=0.95$

Figure 8. The relative efficiency of $\hat{\mathcal{R}}_{U M V U}$ given in (15).

observations based on ordinary records is increased namely that $n^{\prime}>N$ and $m^{\prime}>M$, which is not economical. So, we prefer $\hat{\mathcal{R}}_{U M V U}^{(R R S S)}$ as a efficient estimator.

-Bayesian estimator: From (12), it can be easily shown that the $\operatorname{MSE}\left(\hat{\mathcal{R}}_{B}, \mathcal{R}\right)$ does not depend on the underlying distribution function of $X$ and $Y$. Once more, we consider GE distribution to compare the $\hat{\mathcal{R}}_{B}$ based 
on two schemes and we use relative efficiency as follow

$$
\mathbf{e}\left(\hat{\mathcal{R}}_{B}^{(R R S S)}, \hat{\mathcal{R}}_{B}^{(R)}\right)=\frac{M S E\left(\hat{\mathcal{R}}_{B}^{(R)}, \mathcal{R}\right)}{M S E\left(\hat{\mathcal{R}}_{B}^{(R R S S)}, \mathcal{R}\right)} .
$$

The value of relative efficiency for Bayesian estimators based on two schemes are plotted in Figure 9. From Figure

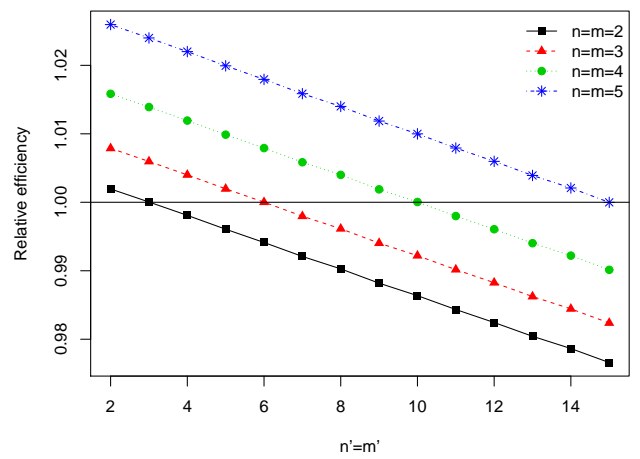

Figure 9. The relative efficiency of $\hat{\mathcal{R}}_{B}$ given in (16).

9, we observe that when $n^{\prime}=m^{\prime} \leq N=M$, we have $\mathbf{e}\left(\hat{\mathcal{R}}_{B}^{(R R S S)}, \hat{\mathcal{R}}_{B}^{(R)}\right)>1$. In this case, the relative efficiency of $\hat{\mathcal{R}}_{B}^{(R R S S)}$ is better than $\hat{\mathcal{R}}_{B}^{(R)}$.

\section{Interval estimation}

A point estimate and CI are closely related. However, CI's provide much more information and are preferred when making inferences. A CI will provide valid result most of the time. In this section, four types of CI's for $\mathcal{R}$ will be derived.

\subsection{CI using Pivotal Quantity}

The $100(1-\gamma) \% \mathrm{CI}$ for $\mathcal{R}$ obtained by using (8) and assuming $W=\frac{\beta N H_{m}^{\prime}}{\alpha M T_{n}^{\prime}}$ as a pivotal quantity as follows

$$
\left[\left(1+\frac{1-\hat{\mathcal{R}}_{M L}}{\hat{\mathcal{R}}_{M L}} F_{1-\frac{\gamma}{2}(2 M, 2 N)}\right)^{-1},\left(1+\frac{1-\hat{\mathcal{R}}_{M L}}{\hat{\mathcal{R}}_{M L}} F_{\frac{\gamma}{2}(2 M, 2 N)}\right)^{-1}\right],
$$

where $F_{(2 M, 2 N)}(\gamma)$ is the $\gamma$-th quantile of F-distribution with $2 M$ and $2 N$ degrees of freedom.

\subsection{Asymptotic CI}

If we consider the number of observed data sufficiently large and $n, m \rightarrow \infty$, we have $\left(\hat{\alpha}_{M L}-\alpha\right) \stackrel{d}{\longrightarrow} N\left(0, \sigma_{1}^{2}\right)$ and $\left(\hat{\beta}_{M L}-\beta\right) \stackrel{d}{\longrightarrow} N\left(0, \sigma_{2}^{2}\right)$ where $(\stackrel{d}{\longrightarrow})$ denotes convergence in distribution. $\sigma_{1}^{2}$ and $\sigma_{2}^{2}$ are the asymptotic variances given by $\sigma_{1}^{2}=-1 / E\left(\frac{\partial^{2}}{\partial \alpha^{2}} \log L(\alpha, \mathbf{U})\right)$ and $\sigma_{2}^{2}=-1 / E\left(\frac{\partial^{2}}{\partial \beta^{2}} \log L(\beta, \mathbf{S})\right)$ with $L$ given in (3). It 
is easy to show that $\sigma_{1}^{2}=\frac{\alpha^{2}}{N}$ and $\sigma_{2}^{2}=\frac{\beta^{2}}{M}$.

Let n,m are sufficiently large in such a way that, $\frac{M}{N} \longrightarrow p$, where $p \in(0,1)$, it follows that

$$
\sqrt{N}\left(\begin{array}{c}
\hat{\alpha}-\alpha \\
\hat{\beta}-\beta
\end{array}\right) \stackrel{d}{\longrightarrow} N_{2}\left(\left(\begin{array}{c}
0 \\
0
\end{array}\right),\left(\begin{array}{cc}
\alpha^{2} & 0 \\
0 & \frac{\beta^{2}}{p}
\end{array}\right)\right) .
$$

By taking $h\left(t_{1}, t_{2}\right)=\frac{t_{2}}{t_{1}+t_{2}}$ and using multivariate delta method (see [29], pp. 5-6) we have

$$
\left(\hat{\mathcal{R}}_{M L}-\mathcal{R}\right) \stackrel{d}{\longrightarrow} N\left(0, \sigma^{2}\right),
$$

where

$$
\sigma=\frac{\alpha \beta}{(\alpha+\beta)^{2}} \sqrt{\frac{1}{N}\left(1+\frac{1}{p}\right)}
$$

based on this asymptotic results and applying the Slutsky's Theorem, a $100(1-\gamma) \%$ asymptotic CI for $\mathcal{R}$ is given by

$$
\left(\hat{\mathcal{R}}_{M L}-Z_{1-\frac{\gamma}{2}} \hat{\sigma}, \hat{\mathcal{R}}_{M L}+Z_{1-\frac{\gamma}{2}} \hat{\sigma}\right) .
$$

Let $Z_{\gamma}$ is the $\gamma$-th quantile of the distribution of standard normal distribution and $\hat{\sigma}$ is obtained by substituting $\hat{\alpha}_{M L}$ and $\hat{\beta}_{M L}$ instead of $\alpha$ and $\beta$ in (18), respectively.

\subsection{Bayesian intervals}

By using the fact that $\frac{\left(\mu_{1}+T_{n}^{\prime}\right)\left(\lambda_{2}+M\right) \alpha}{\left(\mu_{2}+H_{m}^{\prime}\right)\left(\lambda_{1}+N\right) \beta} \mid(\mathbf{r}, \mathbf{s}) \sim F\left(2\left(\lambda_{1}+N\right), 2\left(\lambda_{2}+M\right)\right)$, a $100(1-\gamma) \%$ Bayesian credible interval $(\mathrm{BCI})$ for $\mathcal{R}$ is given by

$$
\left[\left(1+A F_{1-\frac{\gamma}{2}}\right)^{-1},\left(1+A F_{\frac{\gamma}{2}}\right)^{-1}\right]
$$

where $A=\left(\frac{\mu_{2}+H_{m}^{\prime}}{\mu_{1}+T_{n}^{\prime}}\right)\left(\frac{\lambda_{1}+N}{\lambda_{2}+M}\right)$.

We can use the Markov Chain Monte Carlo (MCMC) method proposed by Chen and Shao [9] to compute the HPD interval for $\mathcal{R}$. The construction of HPD intervals requires to find the set,

$$
C\left(\pi_{\alpha}\right)=\left\{\theta: \pi(\theta \mid D) \geq \pi_{\alpha}\right\},
$$

where $\mathrm{D}$ denotes data and $\pi_{\alpha}$ is the largest constant such that $p\left(\theta \in C\left(\pi_{\alpha}\right)\right) \geq 1-\alpha$. (see [6] and [22] for more details).

\subsection{Parametric Bootstrap CI's}

We present some bootstrap intervals for comparison. It is important to know that all inference procedures in this paper depend only on the $T_{n}^{\prime}$ and $H_{m}^{\prime}$ as given in (6) and (7), respectively. So, we use the parametric bootstrap. There are several bootstrap confidence intervals (see [10]) where we have considered percentile, bootstrap-t and basic bootstrap CI's among them. In the following, we will describe the bootstrapping procedure.

Step 1. Calculate $\hat{\alpha}, \hat{\beta}$ and $\hat{\mathcal{R}}_{M L}$ from (6), (7) and (8), respectively by considering independent observed samples $u_{i}, s_{j}$ released from $\operatorname{Gamma}(i, \alpha), i=1, \ldots, n$ and $\operatorname{Gamma}(j, \beta), j=1, \ldots, m$, respectively.

Step 2. Generate $u_{i}^{*} \sim \operatorname{Gamma}(i, \hat{\alpha})$ and $s_{j}^{*} \sim \operatorname{Gamma}(j, \hat{\beta})$ to calculate $\hat{\alpha}^{*}, \hat{\beta}^{*}$ and then $\hat{\mathcal{R}}_{M L}^{*}$.

Step 3. Repeat step 2, B times $(b=1, \ldots, B)$ to obtain $\hat{\mathcal{R}}_{(B) M L}^{*}$. 
4.4.1. Bootstrap-t CI based on the asymptotic standard deviation $\sigma$ : Repeat step 2 for $b=1 \ldots, B$, to drive $\hat{\sigma}^{*}$ in (18) with $\hat{\alpha}^{*}, \hat{\beta}^{*}$ instead of $\hat{\alpha}, \hat{\beta}$, respectively. Let $Z^{*}=\left(Z_{(1)}^{*}, \ldots, Z_{(B)}^{*}\right)^{\top}$ where

$$
z_{(b)}^{*}=\frac{\hat{\mathcal{R}}_{(b) M L}^{*}-\hat{\mathcal{R}}_{M L}}{\hat{\sigma}_{(b)}^{*}}, \quad b=1, \ldots, B
$$

then the $100(1-\gamma) \%$ bootstrap-t $\mathrm{CI}$ for $\mathcal{R}$ is given by

$$
\left(\hat{\mathcal{R}}_{M L}-z_{1-\frac{\gamma}{2}}^{*} \hat{\sigma}, \hat{\mathcal{R}}_{M L}-z_{\frac{\gamma}{2}}^{*} \hat{\sigma}\right) .
$$

where $Z_{\gamma}^{*}$ is the $\gamma$-th quantile of $Z^{*}$.

Refer to [22] for algorithms of Percentile CI, Basic bootstrap CI and Bootstrap-t CI based on the asymptotic standard deviation $\sigma$.

\section{Simulation Study}

In this section, we consider some simulations to compare the performance of the various intervals for $\mathcal{R}$ presented in this paper. In our simulation design, we have used all combinations of $n=3,5,7, m=3,5,7$, $\mathcal{R}=(0.1,0.3,0.5,0.7,0.9)$, and $\beta=0.3$. In each combination, 10000 samples are simulated. We have used $B=1000$ and have considered $B^{\prime}=25$ samples for the bootstrap variance estimate. Also, we consider the prior distribution of both $\alpha$ and $\beta$ to be $\operatorname{Gamma}(2,4)$. We generate the following CI's, and the results of our simulation are given in Tables 1 and 2 .

Perc: Percentile CI

Boot-t1: Bootstrap-t CI based on the asymptotic standard deviation $\sigma$

Boot-t2: Bootstrap-t CI based on bootstrap variance estimate

Basic: the basic bootstrap CI

MLE: CI based on pivotal quantity

AMLE: Approximate CI

Bayes: Bayesian CI

HPD: The approximate HPD interval.

According to the results presented in Tables 1 and 2, we observe the following points:

- As we expected, the EL of all CI's decreases when the sample size increases.

- It appears that the maximum of the EL occurs at $\mathcal{R}=0.5$ and the EL are very small for the extreme values of $\mathcal{R}$, thus the obtained results of simulation study confirm the result of the section 2 .

- The Boot-t1 CI works well compared to the Boot-t2, especially in large samples sizes.

- The CP of the exact CI obtained based on the pivotal quantity and the percentile CI are very close to the true nominal confidence level $\gamma$. However, the AMLE CI outperforms the other bootstrap CI's with respect to EL. Consequently, by considering the two criteria, i.e., the CP and EL, we observe that percentile CI and the MLE CI are better than other CI's.

- As we expected, the HPD CI is better than Bayesian CI. Since the EL of HPD CI is smaller than the EL of Bayesian CI for all $n$ and $m$ combinations, and the CP of HPD CI is more than the CP of Bayesian CI for extreme values of $\mathcal{R}$.

Also, according to the results presented in [22], we observe that when $\gamma=0.1$ the CP and EL are significantly lower than when $\gamma=0.05$.

\subsection{Comparison of interval estimations based on RRSS scheme with ordinary records}

Salehi et al. [27], have compared the two sampling schemes in generating record-breaking data. They have obtained various point estimators for some important parameters of the PHR models based on two sampling schemes and 
Table 1. The CP and EL of $\mathcal{R}$ for $\gamma=0.05$.

\begin{tabular}{|c|c|c|c|c|c|c|c|c|c|c|c|}
\hline \multirow[b]{2}{*}{ Row } & \multirow[b]{2}{*}{$\mathcal{R}$} & \multirow[b]{2}{*}{$n$} & \multirow[b]{2}{*}{$m$} & \multicolumn{2}{|c|}{ perc } & \multicolumn{2}{|c|}{ Boot-t1 } & \multicolumn{2}{|c|}{ Boot-t2 } & \multicolumn{2}{|c|}{ Basic } \\
\hline & & & & $\mathrm{CP}$ & EL & CP & EL & $\mathrm{CP}$ & EL & $\mathrm{CP}$ & EL \\
\hline 1 & 0.1 & 3 & 3 & 0.947 & 0.244 & 0.843 & 0.201 & 0.881 & 0.218 & 0.826 & 0.243 \\
\hline 2 & 0.1 & 3 & 5 & 0.937 & 0.176 & 0.823 & 0.167 & 0.846 & 0.173 & 0.840 & 0.176 \\
\hline 3 & 0.1 & 3 & 7 & 0.922 & 0.156 & 0.811 & 0.156 & 0.829 & 0.161 & 0.841 & 0.155 \\
\hline 4 & 0.1 & 5 & 3 & 0.939 & 0.217 & 0.901 & 0.194 & 0.930 & 0.210 & 0.866 & 0.216 \\
\hline 5 & 0.1 & 5 & 5 & 0.949 & 0.140 & 0.892 & 0.147 & 0.908 & 0.155 & 0.886 & 0.139 \\
\hline 6 & 0.1 & 5 & 7 & 0.945 & 0.116 & 0.885 & 0.127 & 0.896 & 0.134 & 0.891 & 0.116 \\
\hline 7 & 0.1 & 7 & 3 & 0.929 & 0.206 & 0.917 & 0.186 & 0.944 & 0.203 & 0.877 & 0.205 \\
\hline 8 & 0.1 & 7 & 5 & 0.949 & 0.124 & 0.910 & 0.123 & 0.928 & 0.132 & 0.893 & 0.123 \\
\hline 9 & 0.1 & 7 & 7 & 0.949 & 0.098 & 0.907 & 0.101 & 0.923 & 0.107 & 0.904 & 0.098 \\
\hline 10 & 0.3 & 3 & 3 & 0.947 & 0.449 & 0.883 & 0.532 & 0.907 & 0.539 & 0.821 & 0.447 \\
\hline 11 & 0.3 & 3 & 5 & 0.937 & 0.366 & 0.869 & 0.445 & 0.882 & 0.451 & 0.845 & 0.364 \\
\hline 12 & 0.3 & 3 & 7 & 0.923 & 0.337 & 0.854 & 0.418 & 0.864 & 0.424 & 0.854 & 0.336 \\
\hline 13 & 0.3 & 5 & 3 & 0.939 & 0.399 & 0.917 & 0.460 & 0.931 & 0.475 & 0.866 & 0.398 \\
\hline 14 & 0.3 & 5 & 5 & 0.949 & 0.294 & 0.918 & 0.329 & 0.930 & 0.340 & 0.894 & 0.293 \\
\hline 15 & 0.3 & 5 & 7 & 0.945 & 0.255 & 0.911 & 0.285 & 0.921 & 0.295 & 0.902 & 0.254 \\
\hline 16 & 0.3 & 7 & 3 & 0.928 & 0.380 & 0.918 & 0.421 & 0.930 & 0.441 & 0.874 & 0.378 \\
\hline 17 & 0.3 & 7 & 5 & 0.949 & 0.263 & 0.929 & 0.280 & 0.941 & 0.292 & 0.902 & 0.261 \\
\hline 18 & 0.3 & 7 & 7 & 0.949 & 0.217 & 0.927 & 0.230 & 0.937 & 0.240 & 0.914 & 0.216 \\
\hline 19 & 0.5 & 3 & 3 & 0.947 & 0.501 & 0.893 & 0.657 & 0.910 & 0.650 & 0.819 & 0.499 \\
\hline 20 & 0.5 & 3 & 5 & 0.937 & 0.433 & 0.897 & 0.542 & 0.909 & 0.549 & 0.850 & 0.431 \\
\hline 21 & 0.5 & 3 & 7 & 0.922 & 0.407 & 0.891 & 0.502 & 0.898 & 0.514 & 0.857 & 0.406 \\
\hline 22 & 0.5 & 5 & 3 & 0.939 & 0.433 & 0.903 & 0.543 & 0.915 & 0.549 & 0.859 & 0.432 \\
\hline 23 & 0.5 & 5 & 5 & 0.949 & 0.339 & 0.923 & 0.385 & 0.933 & 0.396 & 0.892 & 0.338 \\
\hline 24 & 0.5 & 5 & 7 & 0.946 & 0.301 & 0.929 & 0.333 & 0.938 & 0.344 & 0.905 & 0.300 \\
\hline 25 & 0.5 & 7 & 3 & 0.928 & 0.408 & 0.896 & 0.502 & 0.904 & 0.513 & 0.864 & 0.406 \\
\hline 26 & 0.5 & 7 & 5 & 0.949 & 0.301 & 0.928 & 0.332 & 0.936 & 0.343 & 0.906 & 0.300 \\
\hline 27 & 0.5 & 7 & 7 & 0.949 & 0.254 & 0.934 & 0.272 & 0.943 & 0.282 & 0.917 & 0.253 \\
\hline 28 & 0.7 & 3 & 3 & 0.947 & 0.447 & 0.884 & 0.530 & 0.907 & 0.538 & 0.822 & 0.445 \\
\hline 29 & 0.7 & 3 & 5 & 0.937 & 0.399 & 0.907 & 0.459 & 0.925 & 0.474 & 0.857 & 0.397 \\
\hline 30 & 0.7 & 3 & 7 & 0.923 & 0.380 & 0.912 & 0.422 & 0.926 & 0.442 & 0.871 & 0.378 \\
\hline 31 & 0.7 & 5 & 3 & 0.939 & 0.365 & 0.870 & 0.444 & 0.885 & 0.450 & 0.852 & 0.364 \\
\hline 32 & 0.7 & 5 & 5 & 0.949 & 0.294 & 0.916 & 0.328 & 0.928 & 0.339 & 0.889 & 0.293 \\
\hline 33 & 0.7 & 5 & 7 & 0.945 & 0.263 & 0.931 & 0.280 & 0.942 & 0.293 & 0.910 & 0.262 \\
\hline 34 & 0.7 & 7 & 3 & 0.928 & 0.338 & 0.866 & 0.418 & 0.875 & 0.424 & 0.865 & 0.336 \\
\hline 35 & 0.7 & 7 & 5 & 0.949 & 0.256 & 0.913 & 0.284 & 0.924 & 0.295 & 0.902 & 0.255 \\
\hline 36 & 0.7 & 7 & 7 & 0.949 & 0.217 & 0.930 & 0.230 & 0.941 & 0.240 & 0.915 & 0.216 \\
\hline 37 & 0.9 & 3 & 3 & 0.947 & 0.243 & 0.840 & 0.201 & 0.880 & 0.217 & 0.822 & 0.241 \\
\hline 38 & 0.9 & 3 & 5 & 0.937 & 0.216 & 0.892 & 0.194 & 0.925 & 0.210 & 0.858 & 0.215 \\
\hline 39 & 0.9 & 3 & 7 & 0.922 & 0.206 & 0.915 & 0.187 & 0.943 & 0.204 & 0.874 & 0.205 \\
\hline 40 & 0.9 & 5 & 3 & 0.939 & 0.176 & 0.827 & 0.166 & 0.848 & 0.173 & 0.839 & 0.175 \\
\hline 41 & 0.9 & 5 & 5 & 0.949 & 0.140 & 0.888 & 0.147 & 0.908 & 0.155 & 0.878 & 0.139 \\
\hline 42 & 0.9 & 5 & 7 & 0.945 & 0.125 & 0.920 & 0.124 & 0.935 & 0.132 & 0.901 & 0.124 \\
\hline 43 & 0.9 & 7 & 3 & 0.928 & 0.156 & 0.821 & 0.157 & 0.839 & 0.161 & 0.850 & 0.156 \\
\hline 44 & 0.9 & 7 & 5 & 0.949 & 0.117 & 0.889 & 0.127 & 0.902 & 0.134 & 0.896 & 0.116 \\
\hline 45 & 0.9 & 7 & 7 & 0.949 & 0.099 & 0.913 & 0.101 & 0.925 & 0.107 & 0.907 & 0.098 \\
\hline
\end{tabular}

have compared them with concerning mean squared error and Pitman measure of closeness criteria. It is observed in most of the situations that estimators derived from RRSS scheme are more efficient than their counterparts derived from record sampling scheme.

Baklizi [4] has considered the Likelihood and Bayesian estimation of the stress-strength reliability based on lower record values from the GE distribution. He has achieved exact and approximate confidence intervals and has compared the performance of the intervals by simulation studies. As a special case of the PRHR model, we consider GE distribution to compare the interval estimations based on ordinary records (see tables 1 and 2 of [4]) with those obtained by the RRSS approach. We considered a relative criterion, namely $\delta=\frac{E L^{(R)}}{E L^{(R R S S)}}$.

Which $E L^{(R)}$ and $E L^{(R R S S)}$ indicate expected lengths for the CI based on ordinary record and the RRSS approach, respectively.

In fact, when $\delta>1$, it yields that the CI based on RRSS performs better than the CI based on ordinary record. For the GE distribution, we have derived the values of $\delta$ for some choices of $m$ and $n$ that presented in [4]. From Table 3 and 4 , we observe that $\delta$ is greater than one in all cases.

Therefore, as we expected, the RRSS sampling scheme in PRHR models is better than ordinary records. 
Table 2. continue

\begin{tabular}{|c|c|c|c|c|c|c|c|c|c|c|c|}
\hline \multirow[b]{2}{*}{ Row } & \multirow[b]{2}{*}{$\mathcal{R}$} & \multirow[b]{2}{*}{$n$} & \multirow[b]{2}{*}{$m$} & \multicolumn{2}{|c|}{ MLE } & \multicolumn{2}{|c|}{ AMLE } & \multicolumn{2}{|c|}{ Bayes } & \multicolumn{2}{|c|}{ HPD } \\
\hline & & & & $\mathrm{CP}$ & EL & $\mathrm{CP}$ & EL & $\mathrm{CP}$ & EL & $\mathrm{CP}$ & EL \\
\hline 1 & 0.1 & 3 & 3 & 0.949 & 0.244 & 0.787 & 0.144 & 0.773 & 0.308 & 0.853 & 0.293 \\
\hline 2 & 0.1 & 3 & 5 & 0.938 & 0.177 & 0.624 & 0.080 & 0.758 & 0.255 & 0.860 & 0.240 \\
\hline 3 & 0.1 & 3 & 7 & 0.922 & 0.156 & 0.533 & 0.060 & 0.749 & 0.233 & 0.857 & 0.219 \\
\hline 4 & 0.1 & 5 & 3 & 0.940 & 0.217 & 0.710 & 0.099 & 0.867 & 0.221 & 0.906 & 0.212 \\
\hline 5 & 0.1 & 5 & 5 & 0.951 & 0.140 & 0.648 & 0.063 & 0.856 & 0.166 & 0.900 & 0.160 \\
\hline 6 & 0.1 & 5 & 7 & 0.947 & 0.116 & 0.553 & 0.044 & 0.856 & 0.144 & 0.903 & 0.138 \\
\hline 7 & 0.1 & 7 & 3 & 0.928 & 0.206 & 0.645 & 0.079 & 0.904 & 0.188 & 0.924 & 0.183 \\
\hline 8 & 0.1 & 7 & 5 & 0.949 & 0.124 & 0.572 & 0.047 & 0.905 & 0.133 & 0.917 & 0.130 \\
\hline 9 & 0.1 & 7 & 7 & 0.950 & 0.099 & 0.553 & 0.037 & 0.894 & 0.111 & 0.927 & 0.107 \\
\hline 10 & 0.3 & 3 & 3 & 0.949 & 0.449 & 0.708 & 0.264 & 0.954 & 0.414 & 0.939 & 0.403 \\
\hline 11 & 0.3 & 3 & 5 & 0.938 & 0.366 & 0.586 & 0.166 & 0.961 & 0.356 & 0.957 & 0.345 \\
\hline 12 & 0.3 & 3 & 7 & 0.922 & 0.337 & 0.511 & 0.129 & 0.971 & 0.331 & 0.963 & 0.320 \\
\hline 13 & 0.3 & 5 & 3 & 0.940 & 0.400 & 0.626 & 0.181 & 0.945 & 0.356 & 0.927 & 0.349 \\
\hline 14 & 0.3 & 5 & 5 & 0.951 & 0.294 & 0.609 & 0.132 & 0.951 & 0.284 & 0.942 & 0.278 \\
\hline 15 & 0.3 & 5 & 7 & 0.947 & 0.255 & 0.534 & 0.097 & 0.957 & 0.251 & 0.946 & 0.245 \\
\hline 16 & 0.3 & 7 & 3 & 0.928 & 0.380 & 0.561 & 0.145 & 0.943 & 0.332 & 0.927 & 0.327 \\
\hline 17 & 0.3 & 7 & 5 & 0.949 & 0.263 & 0.544 & 0.100 & 0.948 & 0.251 & 0.937 & 0.247 \\
\hline 18 & 0.3 & 7 & 7 & 0.950 & 0.217 & 0.534 & 0.082 & 0.952 & 0.213 & 0.945 & 0.209 \\
\hline 19 & 0.5 & 3 & 3 & 0.949 & 0.501 & 0.689 & 0.295 & 0.949 & 0.446 & 0.920 & 0.437 \\
\hline 20 & 0.5 & 3 & 5 & 0.938 & 0.433 & 0.587 & 0.196 & 0.946 & 0.392 & 0.923 & 0.385 \\
\hline 21 & 0.5 & 3 & 7 & 0.922 & 0.408 & 0.517 & 0.156 & 0.944 & 0.368 & 0.918 & 0.361 \\
\hline 22 & 0.5 & 5 & 3 & 0.940 & 0.433 & 0.590 & 0.197 & 0.951 & 0.392 & 0.922 & 0.385 \\
\hline 23 & 0.5 & 5 & 5 & 0.951 & 0.339 & 0.598 & 0.153 & 0.950 & 0.321 & 0.932 & 0.316 \\
\hline 24 & 0.5 & 5 & 7 & 0.947 & 0.301 & 0.533 & 0.114 & 0.948 & 0.287 & 0.932 & 0.283 \\
\hline 25 & 0.5 & 7 & 3 & 0.928 & 0.408 & 0.527 & 0.156 & 0.947 & 0.368 & 0.921 & 0.361 \\
\hline 26 & 0.5 & 7 & 5 & 0.949 & 0.301 & 0.528 & 0.114 & 0.949 & 0.287 & 0.931 & 0.282 \\
\hline 27 & 0.5 & 7 & 7 & 0.950 & 0.254 & 0.527 & 0.096 & 0.948 & 0.246 & 0.937 & 0.243 \\
\hline 28 & 0.7 & 3 & 3 & 0.949 & 0.448 & 0.706 & 0.263 & 0.936 & 0.401 & 0.911 & 0.390 \\
\hline 29 & 0.7 & 3 & 5 & 0.938 & 0.399 & 0.622 & 0.181 & 0.922 & 0.355 & 0.905 & 0.349 \\
\hline 30 & 0.7 & 3 & 7 & 0.922 & 0.380 & 0.548 & 0.145 & 0.912 & 0.334 & 0.896 & 0.329 \\
\hline 31 & 0.7 & 5 & 3 & 0.940 & 0.366 & 0.592 & 0.166 & 0.950 & 0.340 & 0.924 & 0.329 \\
\hline 32 & 0.7 & 5 & 5 & 0.951 & 0.294 & 0.608 & 0.132 & 0.944 & 0.280 & 0.930 & 0.273 \\
\hline 33 & 0.7 & 5 & 7 & 0.947 & 0.263 & 0.544 & 0.100 & 0.940 & 0.251 & 0.931 & 0.246 \\
\hline 34 & 0.7 & 7 & 3 & 0.928 & 0.338 & 0.522 & 0.129 & 0.947 & 0.315 & 0.921 & 0.302 \\
\hline 35 & 0.7 & 7 & 5 & 0.949 & 0.256 & 0.531 & 0.097 & 0.948 & 0.247 & 0.932 & 0.240 \\
\hline 36 & 0.7 & 7 & 7 & 0.950 & 0.218 & 0.533 & 0.083 & 0.947 & 0.212 & 0.937 & 0.208 \\
\hline 37 & 0.9 & 3 & 3 & 0.949 & 0.242 & 0.784 & 0.143 & 0.927 & 0.221 & 0.927 & 0.207 \\
\hline 38 & 0.9 & 3 & 5 & 0.938 & 0.216 & 0.702 & 0.098 & 0.905 & 0.192 & 0.919 & 0.184 \\
\hline 39 & 0.9 & 3 & 7 & 0.922 & 0.206 & 0.630 & 0.080 & 0.890 & 0.180 & 0.905 & 0.173 \\
\hline 40 & 0.9 & 5 & 3 & 0.940 & 0.176 & 0.632 & 0.080 & 0.948 & 0.168 & 0.932 & 0.156 \\
\hline 41 & 0.9 & 5 & 5 & 0.951 & 0.140 & 0.646 & 0.063 & 0.942 & 0.135 & 0.939 & 0.129 \\
\hline 42 & 0.9 & 5 & 7 & 0.947 & 0.125 & 0.578 & 0.048 & 0.935 & 0.120 & 0.937 & 0.116 \\
\hline 43 & 0.9 & 7 & 3 & 0.928 & 0.157 & 0.548 & 0.060 & 0.947 & 0.150 & 0.924 & 0.138 \\
\hline 44 & 0.9 & 7 & 5 & 0.949 & 0.117 & 0.554 & 0.045 & 0.947 & 0.115 & 0.938 & 0.109 \\
\hline 45 & 0.9 & 7 & 7 & 0.950 & 0.099 & 0.551 & 0.038 & 0.945 & 0.097 & 0.942 & 0.094 \\
\hline
\end{tabular}

\section{Data analysis}

To demonstrate the procedures proposed in preceding sections, we analyze a real data set. Here, we consider a data set on the time of successive failures of the air conditioning system of each number of a fleet of Boeing 720 jet airplanes. The mentioned data are available in [19]. We randomly select two airplanes to study the stress-strength reliability. Here, we consider plans 7908 and 8045 to see whether the GE distribution is adequate to fit on this data sets or not. We used the Kolomogrove- Smirnov (K-S) test, and we see that corresponding P-values of the K-S test for the observations $X$ and $Y$ are 0.372 and 0.667 , respectively, which support the adequacy of the GE distribution. It should be noted that any other airplane that support the adequacy of the specific distribution can be selected for study. The extracted values of lower RRSS from this data set according to Diagram 1 are presented in Table 5. From Table 5, we see that each of the numbers 7908 and 8045 of Boeing 720 jet airplanes has a lower RRSS of size four from air condition data. To study the $\mathcal{R}=: P(X<Y)$, let the $X$-variable represent the stress which is demonstrated with the lower RRSS values of number 7908 airplane as $\mathbf{u}=(413,9,34,7)$ and $Y$-variable represent the strength which is demonstrated with the lower RRSS values of number 8045 airplane as $\mathbf{s}=(102,14,32,14)$ $(n=m=4)$.

In order to check the performance of the point and interval estimations, the bootstrap method is employed in order to re-sample the data and so provide more observations for the estimators. To this purpose, 200 bootstrap samples are generated from the original data sets, and then the RRSS's are extracted. Based on the obtained RRSS's, we get the $\hat{\mathcal{R}}_{M L}=0.655$ and $\hat{\mathcal{R}}_{U M V U}=0.663$ as the point estimation of the stress-strength reliability parameter. The 
Table 3. The values of $\delta$ for some values of $m$ and $n$ when $\gamma=0.05$

\begin{tabular}{|c|c|c|c|c|c|c|c|c|c|c|c|c|c|c|c|c|c|c|}
\hline \multirow[b]{2}{*}{ Row } & \multirow[b]{2}{*}{$n$} & \multirow[b]{2}{*}{$m$} & \multirow[b]{2}{*}{$\mathcal{R}$} & \multicolumn{3}{|c|}{ MLE } & \multicolumn{3}{|c|}{ Perc } & \multicolumn{3}{|c|}{ Boot-t1 } & \multicolumn{3}{|c|}{ Boot-t2 } & \multicolumn{3}{|c|}{ HPD } \\
\hline & & & & $\delta$ & $C P^{R}$ & $C P^{R R S S}$ & $\delta$ & $C P^{R}$ & $C P^{R R S S}$ & $\delta$ & $C P^{R}$ & $C P^{R R S S}$ & $\delta$ & $C P^{R}$ & $C P^{R R S S}$ & $\delta$ & $C P^{R}$ & $C P^{R R S S}$ \\
\hline 1 & 5 & 5 & 0.1 & 1.576 & 0.832 & 0.920 & 2.223 & 0.912 & 0.904 & 2.959 & 0.881 & 0.923 & 3.101 & 0.883 & 0.919 & 1.879 & 0.877 & 0.902 \\
\hline 2 & 5 & 5 & 0.3 & 1.476 & 0.806 & 0.920 & 1.701 & 0.919 & 0.902 & 2.599 & 0.891 & 0.929 & 2.315 & 0.888 & 0.932 & 1.679 & 0.865 & 0.895 \\
\hline 3 & 5 & 5 & 0.5 & 1.509 & 0.819 & 0.920 & 1.593 & 0.918 & 0.898 & 2.621 & 0.902 & 0.930 & 2.175 & 0.897 & 0.938 & 1.700 & 0.881 & 0.908 \\
\hline 4 & 5 & 10 & 0.1 & 1.847 & 0.886 & 0.888 & 3.049 & 0.845 & 0.870 & 2.578 & 0.932 & 0.884 & 2.143 & 0.949 & 0.969 & 2.176 & 0.900 & 0.925 \\
\hline 5 & 5 & 10 & 0.3 & 1.669 & 0.846 & 0.888 & 2.078 & 0.833 & 0.873 & 2.539 & 0.912 & 0.892 & 1.881 & 0.909 & 0.968 & 1.991 & 0.887 & 0.909 \\
\hline 6 & 5 & 10 & 0.5 & 1.583 & 0.814 & 0.888 & 1.691 & 0.835 & 0.869 & 2.593 & 0.837 & 0.897 & 1.842 & 0.850 & 0.966 & 1.925 & 0.889 & 0.907 \\
\hline 7 & 5 & 15 & 0.1 & 1.962 & 0.910 & 0.907 & 3.292 & 0.779 & 0.881 & 2.514 & 0.938 & 0.905 & 1.721 & 0.943 & 0.995 & 2.248 & 0.899 & 0.921 \\
\hline 8 & 5 & 15 & 0.3 & 1.726 & 0.833 & 0.907 & 2.124 & 0.774 & 0.880 & 2.504 & 0.862 & 0.916 & 1.553 & 0.854 & 0.995 & 2.027 & 0.865 & 0.912 \\
\hline 9 & 5 & 15 & 0.5 & 1.605 & 0.810 & 0.907 & 1.667 & 0.783 & 0.878 & 2.572 & 0.811 & 0.917 & 1.539 & 0.808 & 0.991 & 1.952 & 0.886 & 0.902 \\
\hline 10 & 10 & 5 & 0.1 & 1.897 & 0.868 & 0.898 & 1.560 & 0.887 & 0.892 & 3.152 & 0.820 & 0.907 & 2.476 & 0.763 & 0.970 & 1.877 & 0.899 & 0.902 \\
\hline 11 & 10 & 5 & 0.3 & 2.004 & 0.886 & 0.898 & 1.570 & 0.893 & 0.893 & 2.953 & 0.880 & 0.915 & 2.058 & 0.816 & 0.977 & 1.864 & 0.895 & 0.904 \\
\hline 12 & 10 & 5 & 0.5 & 2.098 & 0.887 & 0.898 & 1.679 & 0.877 & 0.887 & 2.864 & 0.903 & 0.916 & 1.893 & 0.833 & 0.976 & 1.910 & 0.893 & 0.921 \\
\hline 13 & 10 & 10 & 0.1 & 2.217 & 0.903 & 0.897 & 2.580 & 0.933 & 0.908 & 3 & 0.915 & 0.906 & 3.167 & 0.896 & 0.905 & 2.559 & 0.926 & 0.903 \\
\hline 14 & 10 & 10 & 0.3 & 2.167 & 0.889 & 0.897 & 2.237 & 0.918 & 0.907 & 2.894 & 0.936 & 0.908 & 2.796 & 0.916 & 0.915 & 2.477 & 0.901 & 0.901 \\
\hline 15 & 10 & 10 & 0.5 & 2.158 & 0.858 & 0.897 & 2.109 & 0.910 & 0.906 & 2.889 & 0.914 & 0.910 & 2.663 & 0.911 & 0.919 & 2.478 & 0.907 & 0.910 \\
\hline 16 & 10 & 15 & 0.1 & 2.448 & 0.910 & 0.892 & 3.105 & 0.891 & 0.886 & 3.085 & 0.942 & 0.888 & 2.831 & 0.935 & 0.934 & 2.879 & 0.921 & 0.900 \\
\hline 17 & 10 & 15 & 0.3 & 2.271 & 0.885 & 0.892 & 2.515 & 0.906 & 0.886 & 2.949 & 0.942 & 0.892 & 2.527 & 0.935 & 0.937 & 2.727 & 0.915 & 0.897 \\
\hline 18 & 10 & 15 & 0.5 & 2.215 & 0.876 & 0.892 & 2.266 & 0.900 & 0.883 & 2.951 & 0.910 & 0.895 & 2.454 & 0.921 & & 2.716 & 0.923 & 0.898 \\
\hline 19 & 15 & 5 & 0.1 & 2.330 & 0.891 & 0.897 & 1.413 & 0.841 & 0.883 & 3.291 & 0.772 & 0.901 & 2.059 & 0.710 & 0.992 & 1.869 & 0.888 & 0.903 \\
\hline 20 & 15 & 5 & 0.3 & 2.437 & 0.918 & 0.897 & 1.487 & 0.824 & 0.884 & 3 & 0.814 & 0.904 & 1.731 & 0.739 & 0.996 & 1.855 & 0.885 & 0.904 \\
\hline 21 & 15 & 5 & 0.5 & 2.636 & 0.930 & 0.897 & 1.681 & 0.837 & 0.881 & 2.907 & 0.872 & 0.905 & 1.613 & 0.795 & 0.994 & 1.944 & 0.893 & 0.910 \\
\hline 22 & 15 & 10 & 0.1 & 2.621 & 0.915 & 0.890 & 2.458 & 0.910 & 0.888 & 3.237 & 0.886 & 0.874 & 2.886 & 0.851 & 0.940 & 2.655 & 0.915 & 0.894 \\
\hline 23 & 15 & 10 & 0.3 & 2.594 & 0.922 & 0.890 & 2.269 & 0.923 & 0.886 & 3.103 & 0.932 & 0.876 & 2.561 & 0.891 & 0.947 & 2.634 & 0.920 & 0.896 \\
\hline 24 & 15 & 10 & 0.5 & 2.652 & 0.920 & 0.890 & 2.259 & 0.927 & 0.888 & 3.118 & 0.942 & 0.878 & 2.482 & 0.919 & 0.947 & 2.703 & 0.931 & 0.893 \\
\hline 25 & 15 & 15 & 0.1 & 2.739 & 0.909 & 0.915 & 2.957 & 0.911 & 0.906 & 3.348 & 0.921 & 0.904 & 3.479 & 0.900 & 0.910 & 3.065 & 0.916 & 0.901 \\
\hline 26 & 15 & 15 & 0.3 & 2.679 & 0.913 & 0.915 & 2.660 & 0.927 & 0.905 & 3.271 & 0.945 & 0.904 & 3.205 & 0.926 & 0.912 & 3.048 & 0.932 & 0.901 \\
\hline 27 & 15 & 15 & 0.5 & 2.690 & 0.913 & 0.915 & 2.568 & 0.920 & 0.906 & 3.299 & 0.940 & 0.904 & 3.128 & 0.938 & 0.913 & 3.130 & 0.934 & 0.896 \\
\hline
\end{tabular}

Table 4. The values of $\delta$ for some values of $m$ and $n$ when $\gamma=0.1$.

\begin{tabular}{|c|c|c|c|c|c|c|c|c|c|c|c|c|c|c|c|c|c|c|}
\hline \multirow[b]{2}{*}{ Row } & \multirow[b]{2}{*}{$n$} & \multirow[b]{2}{*}{$m$} & \multirow[b]{2}{*}{$\mathcal{R}$} & \multicolumn{3}{|c|}{ 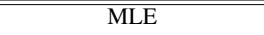 } & \multicolumn{3}{|c|}{ Perc } & \multicolumn{3}{|c|}{ Boot-t1 } & \multicolumn{3}{|c|}{ Boot-t2 } & \multicolumn{3}{|c|}{$\overline{~ H P D}$} \\
\hline & & & & $\delta$ & $C P^{R}$ & $C P^{R R S S}$ & $\delta$ & $C P^{R}$ & $C P^{R R S S}$ & $\delta$ & $C P^{R}$ & $C P^{R R S S}$ & $\delta$ & $C P^{R}$ & $C P^{R R S S}$ & $\delta$ & $C P^{R}$ & $C P^{R R S S}$ \\
\hline 1 & 5 & 5 & 0.1 & 1.565 & 0.774 & 0.920 & 2.086 & 0.846 & 0.904 & 2.620 & 0.841 & 0.923 & 2.756 & 0.836 & 0.919 & 1.853 & 0.802 & 0.902 \\
\hline 2 & 5 & 5 & 0.3 & 1.476 & 0.760 & 0.920 & 1.669 & 0.856 & 0.902 & 2.361 & 0.864 & 0.929 & 2.135 & 0.852 & 0.932 & 1.764 & 0.785 & 0.895 \\
\hline 3 & 5 & 5 & 0.5 & 1.497 & 0.752 & 0.920 & 1.559 & 0.852 & 0.898 & 2.381 & 0.857 & 0.930 & 2.021 & 0.855 & 0.938 & 1.809 & 0.811 & 0.908 \\
\hline 4 & 5 & 10 & 0.1 & 1.891 & 0.815 & 0.888 & 2.942 & 0.736 & 0.870 & 2.511 & 0.895 & 0.884 & 2.062 & 0.900 & 0.969 & 2.303 & 0.817 & 0.925 \\
\hline 5 & 5 & 10 & 0.3 & 1.665 & 0.750 & 0.888 & 2.021 & 0.742 & 0.873 & 2.390 & 0.823 & 0.892 & 1.771 & 0.815 & 0.968 & 2.118 & 0.777 & 0.909 \\
\hline 6 & 5 & 10 & 0.5 & 1.575 & 0.758 & 0.888 & 1.664 & 0.759 & 0.869 & 2.419 & 0.792 & 0.897 & 1.730 & 0.800 & 0.966 & 2.049 & 0.814 & 0.907 \\
\hline 7 & 5 & 15 & 0.1 & 2 & 0.841 & 0.907 & 3.175 & 0.678 & 0.881 & 2.477 & 0.891 & 0.905 & 1.667 & 0.883 & 0.995 & 2.369 & 0.821 & 0.921 \\
\hline 8 & 5 & 15 & 0.3 & 1.721 & 0.755 & 0.907 & 2.077 & 0.665 & 0.880 & 2.387 & 0.785 & 0.916 & 1.483 & 0.772 & 0.995 & 2.196 & 0.773 & 0.912 \\
\hline 9 & 5 & 15 & 0.5 & 1.591 & 0.736 & 0.907 & 1.632 & 0.671 & 0.878 & 2.401 & 0.735 & 0.917 & 1.441 & 0.737 & 0.991 & 2.066 & 0.794 & 0.902 \\
\hline 10 & 10 & 5 & 0.1 & 1.888 & 0.803 & 0.898 & 1.516 & 0.793 & 0.892 & 2.804 & 0.752 & 0.907 & 2.202 & 0.691 & 0.970 & 1.820 & 0.809 & 0.902 \\
\hline 11 & 10 & 5 & 0.3 & 1.975 & 0.823 & 0.898 & 1.544 & 0.793 & 0.893 & 2.641 & 0.799 & 0.915 & 1.859 & 0.744 & 0.977 & 1.927 & 0.808 & 0.904 \\
\hline 12 & 10 & 5 & 0.5 & 2.090 & 0.846 & 0.898 & 1.661 & 0.818 & 0.887 & 2.603 & 0.857 & 0.916 & 1.751 & 0.797 & 0.976 & 2.049 & 0.844 & 0.921 \\
\hline 13 & 10 & 10 & 0.1 & 2.263 & 0.838 & 0.897 & 2.509 & 0.843 & 0.908 & 2.879 & 0.884 & 0.906 & 3 & 0.867 & 0.905 & 2.596 & 0.854 & 0.903 \\
\hline 14 & 10 & 10 & 0.3 & 2.153 & 0.828 & 0.897 & 2.176 & 0.846 & 0.907 & 2.739 & 0.881 & 0.908 & 2.645 & 0.858 & 0.915 & 2.612 & 0.846 & 0.901 \\
\hline 15 & 10 & 10 & 0.5 & 2.174 & 0.828 & 0.897 & 2.071 & 0.847 & 0.906 & 2.766 & 0.882 & 0.910 & 2.573 & 0.872 & 0.919 & 2.691 & 0.862 & 0.910 \\
\hline 16 & 10 & 15 & 0.1 & 2.388 & 0.847 & 0.892 & 2.917 & 0.829 & 0.886 & 2.959 & 0.910 & 0.888 & 2.644 & 0.902 & 0.934 & 2.898 & 0.872 & 0.900 \\
\hline 17 & 10 & 15 & 0.3 & 2.259 & 0.825 & 0.892 & 2.441 & 0.818 & 0.886 & 2.833 & 0.890 & 0.892 & 2.434 & 0.890 & 0.937 & 2.928 & 0.851 & 0.897 \\
\hline 18 & 10 & 15 & 0.5 & 2.195 & 0.815 & 0.892 & 2.203 & 0.817 & 0.883 & 2.830 & 0.855 & 0.895 & 2.358 & 0.861 & 0.938 & 2.946 & 0.852 & 0.898 \\
\hline 19 & 15 & 5 & 0.1 & 2.274 & 0.860 & 0.897 & 1.367 & 0.759 & 0.883 & 2.849 & 0.717 & 0.901 & 1.812 & 0.660 & 0.992 & 1.807 & 0.818 & 0.903 \\
\hline 20 & 15 & 5 & 0.3 & 2.433 & 0.869 & 0.897 & 1.461 & 0.751 & & 2.682 & & & 1.562 & 0.682 & & 1.906 & 0.807 & 0.904 \\
\hline 21 & 15 & 5 & 0.5 & 2.618 & 0.892 & 0.897 & 1.653 & 0.769 & 0.881 & 2.628 & 0.820 & 0.905 & 1.487 & 0.740 & 0.994 & 2.075 & 0.842 & 0.910 \\
\hline 22 & 15 & 10 & 0.1 & 2.592 & 0.861 & 0.890 & 2.320 & 0.839 & 0.888 & 3.061 & 0.837 & 0.874 & 2.672 & 0.803 & 0.940 & 2.673 & 0.858 & 0.894 \\
\hline 23 & 15 & 10 & 0.3 & 2.589 & 0.868 & 0.890 & 2.204 & 0.845 & 0.886 & 2.939 & 0.873 & 0.876 & 2.434 & 0.843 & 0.947 & 2.791 & 0.859 & 0.896 \\
\hline 24 & 15 & 10 & 0.5 & 2.639 & 0.871 & 0.890 & 2.195 & 0.847 & 0.888 & 2.933 & 0.888 & 0.878 & 2.364 & 0.857 & 0.947 & 2.938 & 0.877 & 0.893 \\
\hline 25 & 15 & 15 & 0.1 & 2.744 & 0.874 & 0.915 & 2.872 & 0.852 & 0.906 & 3.282 & 0.908 & 0.904 & 3.375 & 0.895 & 0.910 & 3.289 & 0.876 & 0.901 \\
\hline 26 & 15 & 15 & 0.3 & 2.674 & 0.857 & 0.915 & 2.584 & 0.855 & 0.905 & 3.133 & 0.896 & 0.904 & 3.065 & 0.879 & 0.912 & 3.261 & 0.871 & 0.901 \\
\hline 27 & 15 & 15 & 0.5 & 2.660 & 0.850 & 0.915 & 2.472 & 0.842 & 0.906 & 3.140 & 0.888 & 0.904 & 3.018 & 0.882 & 0.913 & 3.385 & 0.869 & 0.896 \\
\hline
\end{tabular}

corresponding box-plot of the observations of the point estimates are plotted in Figure 10. According to the values of point estimators that are around 0.6 , we expected $\hat{\mathcal{R}}_{M L}$ to have a better efficiency than $\hat{\mathcal{R}}_{U M V U}$. It can be seen that the interquartile range in $\hat{\mathcal{R}}_{M L}$ is less than $\hat{\mathcal{R}}_{U M V U}$. Therefore, as we have already observed in Figure 4 , the performance of $\hat{\mathcal{R}}_{M L}$ is better than that of $\hat{\mathcal{R}}_{U M V U}$ in estimating the middle value of $\mathcal{R}$. For inspecting the CI's, 
Table 5. Extracted lower RRSS values from Air condition data

\begin{tabular}{ccccccccccccc}
\hline & \multicolumn{10}{c}{ plan } & \multicolumn{10}{c}{$c$} \\
\hline 7907 & 7908 & 7909 & 7910 & 7911 & 7912 & 7913 & 7914 & 7915 & 7916 & 7917 & 8044 & 8045 \\
\hline 194 & 413 & 90 & 74 & 55 & 23 & 97 & 50 & 359 & 50 & 130 & 487 & 102 \\
& 9 & & 48 & 56 & 87 & 11 & 22 & 3 & 5 & & 7 & 14 \\
& 34 & & & 33 & 3 & & & & 12 & & 3 & 32 \\
& 7 & & & & 1 & & & & & & & 14 \\
\hline
\end{tabular}

we have replicated the above procedures 1000 times more. The distribution $\operatorname{Gamma}(2,1)$ and $\operatorname{Gamma}(2,2)$ have been applied as the prior pdf for $\alpha$ and $\beta$, respectively. The EL's of the intervals are reported in Table 6.

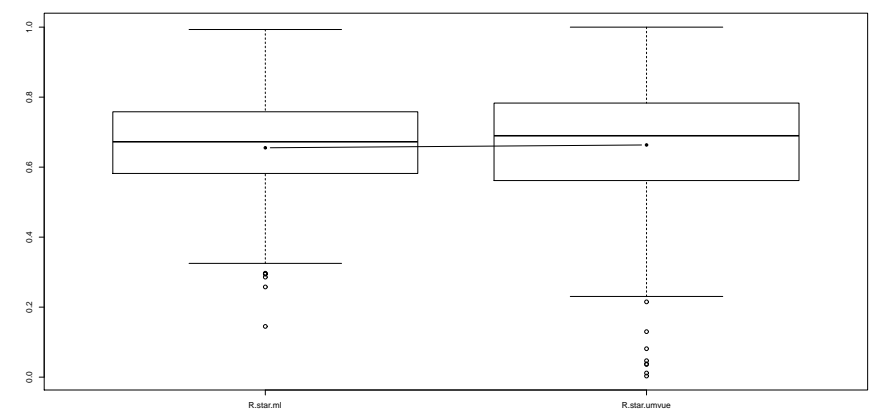

Figure 10. Comparison of $\hat{\mathcal{R}}_{M L}$ and $\hat{\mathcal{R}}_{U M V U}$.

Table 6. The values of EL of $\mathcal{R}$ based on real data set

\begin{tabular}{ccccccccc}
\hline$\gamma$ & Perc & Boot-t1 & Boot-t2 & Basic & MLE & AMLE & Bayes & HPD \\
\hline 0.05 & 0.593 & 0.894 & 0.598 & 0.578 & 0.389 & 0.396 & 0.385 & 0.359 \\
\hline 0.1 & 0.494 & 0.688 & 0.431 & 0.487 & 0.329 & 0.332 & 0.327 & 0.306 \\
\hline
\end{tabular}

According to the results of Table 6, it is observed that like the previous section the EL of Boot-t1 is smaller than Boot-t2. Also, the EL of MLE and AMLE are better than the other CI's, especially in more detail, the MLE CI has the least expected length. As we expected, the HPD CI has a better performance than Bayesian CI. Thus, the obtained result in this section are quite consistent with the results of the preceding section.

\section{Conclusion}

In this paper, we obtained the MLE, UMVUE, as well as the Bayes estimator for stress-strength parameter $\mathcal{R}$ on the basis of lower RRSS from the PRHR model. The MLE and UMVUE are compared with respect to the relative efficiency criterion. It is observed that the MLE exhibits a better performance when $\mathcal{R}$ is close to 0.5, while UMVUE is better for the extreme values of $\mathcal{R}$. Also, we compared these point estimators based on two schemes (RRSS and ordinary record) with respect to the relative efficiency criteria. It is observed that the estimators for $\mathcal{R}$ based on RRSS are seemingly superior to ordinary records. We derived various CI's and compared them via a simulation study. Based on a simulation result and an illustrative example, we observe that the percentile CI and the exact CI of MLE are better than the other CI's. Also, HPD CI has a better performance than Bayesian CI. Finally, we compared the interval estimations based on ordinary records with those obtained by the RRSS approach for the GE distribution. We observe that the CI based on RRSS is better than ordinary records and we also proved that all calculations do not depend on the baseline distribution in the PRHR model. 


\section{Acknowledgement}

The authors are thankful to the editor and referees for careful reading and helpful suggestions.

\section{REFERENCES}

1. B. C. Arnold, N. Balakrishnan, and H. N. Nagaraja, Records, John Wiley and Sons, New York, 1998.

2. A. Asgharzadeh, R. Valiollahi, and M. Z. Raqab, Estimation of $\operatorname{Pr}(Y<X)$ for the two-parameter of generalized exponential records Communications in Statistics-Simulation and Computation, vol. 46, no. 1, pp. 379-394, 2017.

3. W. N. Bailey, Generalized Hyper-geometric Series, University Press, Cambridge, 1935.

4. A. Baklizi, Likelihood and Bayesian estimation of $\operatorname{Pr}(X<Y)$ using lower record values from the generalized exponential distribution, Computational Statistics and Data Analysis, vol. 52, no. 7, pp. 3468-3473, 2008.

5. M. Basirat, S. Baratpour, and J. Ahmadi, On the estimation of the stress-strength parameter using record valued from proportional hazard rate models Communications in Statistics-Theory and Methods. vol. 45, no. 19, pp. 5787-5801, 2016.

6. J. 0. Berger, Statistical Decision Theory and Bayesian Analysis (2nd ed.), New York: Wiley, 1985.

7. G. Casella, and R. L. Berger, Statistical Inference, Pacific Grove, CA: Wadsworth/Brooks Cole, 1990.

8. N. N. Chandra, and D. Roy, Some results on the reversed hazard rate, Probability in the Engineering and Informational Sciences, vol. 15, no. 1, pp. 95-102, 2001.

9. M. -H. Chen, and Q. -M. Shao, Monte Carlo estimation of Bayesian credible and HPD intervals, Journal of Computational and Graphical Statistics, vol. 8, no. 1, pp. 69-92, 1999.

10. B. Efron, and R. Tibshirani, An Introduction to the Bootstrap, Chapman and Hall, New York, 1993.

11. M. Eskandarzadeh, S. Tahmasebi, and M. Afshari, Information measures for record ranked set samples, Ciencia e Natura, vol. 38, no. 2, pp. 554-563, 2016.

12. R. D. Gupta, and A. K. Nanda, Some results on (reversed) hazard rate ordering, Communication in Statistics: Theory and Methods, vol. 30, no. 11, pp. 2447-2458, 2001.

13. R. C. Gupta, P. L. Gupta, and R. D. Gupta, Modeling failure time data by Lehman alternatives, Communication in Statistics: Theory and Methods, vol. 27, no. 4, pp. 887-904, 1998.

14. R. C. Gupta, and R. D. Gupta, Proportional reversed hazard rate model and its applications, Journal of Statistical Planning and Inference vol. 137, no. 11, pp. 3525-3536, 2007.

15. S. Kotz, Y. Lumelskii, and M. Pensky, The Stress-Strength Model and its Generalizations: theory and applications, World Scientific, Singapore, 2003.

16. E. L. Lehmann, and G. Casella, Theory of Point Estimation, Second Edition, Springer Texts in Statistics, New York, 1998.

17. H. A. Muttlak, W. A. Abu-Dayyeh, M. F. Saleh, and E. Al-Sawi, Estimating $\operatorname{Pr}(Y<X)$ using ranked set sampling in case of the exponential distribution, Communication in Statistics: Theory and Methods, vol. 39, no. 10, pp. 1855-1868, 2010.

18. J. Paul, and PY. Thomas, Concomitant record ranked set sampling, Communications in Statistics-Theory and methods, vol. 46, no. 19, pp. 9518-9540, 2017.

19. Proschan Theoretical explanation of observed decreasing failure rate, Technometrics, vol. 5, no. 3, pp. 375-383, 1963.

20. M. Z. Raqab, M.T. Madi, and D. Kundu, Estimation of $\operatorname{Pr}(Y<X)$ for the three-parameter generalized exponential distribution, Communications in Statistics-Theory and Methods, vol. 37, no. 18, pp. 2854-2865, 2008.

21. S. Rezaei, R. Tahmasbi, and M. Mahmoodi, Estimation of $\operatorname{Pr}(Y<X)$ for generalized Pareto distribution, Journal of Statistical Planing and Inference, vol. 140, no. 2, pp. 480-494, 2010.

22. A. Sadeghpour, M. Salehi, and A. Nezakati, Estimation of stress-strength reliability using record ranked set sampling scheme under the generalized exponential distribution, Journal of Statistical Computation and Simulation, vol. 90, no. 1, pp. 51-74, 2020.

23. A. Safariyan, M. Arashi, and R. Arabi Belaghi, Improved point and interval estimation of the stress-strength reliability based on record ranked set sampling scheme, Statistics, vol. 53, no. 1, pp. 101-125, 2019.

24. A. Safariyan, M. Arashi, and R. Arabi Belaghi, Improved estimators for stress-strength reliability using record ranked set sampling scheme, Communications in Statistics - Simulation and Computation, vol. 48, no. 9, pp. 2708-2726, 2019.

25. M. Salehi, and J. Ahmadi, Record ranked set sampling scheme, Metron, vol. 72, no. 3, pp. 351-365, 2014.

26. M. Salehi, and J. Ahmadi, Estimation of stress-strength using record ranked set sampling scheme from the exponential distribution, Filomat, vol. 29, no. 5, pp. 1149-1162, 2015.

27. M. Salehi, and J. Ahmadi, and S. Day, Comparison of two sampling schemes for generating record-breaking data from the proportional hazard rate models, Communications in Statistics- Theory and Methods, vol. 45, no. 13, pp. 3721-3733, 2016.

28. D. Sengupta, and A. K. Nanda, Log-concave and concave distributions in reliability, Naval Res. Logistics Quart. vol. 46, no. 4, pp. 419-433, 1999.

29. L. Wasserman, All of Non-parametric Statistics, Springer, New York, 2006. 